\title{
The birds of Reserva Extrativista Chico Mendes, South Acre, Brazil Aves da Reserva Extrativista Chico Mendes, Sul do Acre, Brasil
}

\author{
Luiz Augusto Macedo Mestre \\ Gregory Thom" \\ Mark Alan Cochrane $e^{\text {III }}$ \\ Jos Barlowiv
}

\begin{abstract}
This paper describes the avifauna sampled at Reserva Extrativista Chico Mendes, Acre, Brazil, during October and November, 2008. We recorded 344 bird species of 17 orders and 57 families through point counts, mist-nets and general observations. The most prevalent families were Tyrannidae, Thamnophilidae and Thraupidae with 53, 36 and 22 species, respectively. We recorded some range restricted, little know, and habitat specialists birds exemplified by Crypturellus atrocapillus, C. strigulosus, Primolius couloni, Aulacorhynchus prasinus, Drymophila devillei, Simoxenops ucayalae, Cnipodectes superrufus, Hemitriccus flammulatus, Percnostola lophotes, Xiphorhynchus chunchotambo, and Conioptilon mcilhennyi. Although we surveyed only during the dry season, the rarefaction curves indicate a satisfactory sampling effort. The data show that the Chico Mendes reserve holds a unique Amazonian bird community, which is influenced by the presence of bamboo and second growth vegetation. The results of this paper reinforce the biological importance of the RESEX and highlight the need for more inventories and bird studies at this isolated and little known region of the Brazilian Amazon.
\end{abstract}

Keywords: Aves. Brazil. Southeast Acre. Southwest Amazon. Reserva Extrativista Chico Mendes.

Resumo: Este estudo descreve a avifauna amostrada na Reserva Extrativista Chico Mendes, Acre, Brasil. Foram registradas por contagem por pontos, redes neblina e observações gerais 344 espécies de aves incluídas em 17 ordens e 57 famílias. As famílias mais representadas foram Tyrannidae, Thamnophilidae e Thraupidae com 53, 36 e 22 espécies, respectivamente. Registramos algumas espécies de distribuição restrita, pouco conhecidas ou especialistas, exemplificadas por Crypturellus atrocapillus, C. strigulosus, Primolius couloni, Aulacorhynchus prasinus, Drymophila devillei, Simoxenops ucayalae, Cnipodectes superrufus, Hemitriccus flammulatus, Xiphorhynchus chunchotambo, Percnostola lophotes e Conioptilon mcilhennyi. Apesar de termos amostrado apenas a estação seca, as curvas cumulativas de espécies indicaram um bom esforço amostral. Os resultados deste estudo mostraram que nesta reserva ocorre uma comunidade de aves característica, influenciada por bambus e florestas secundárias. Nossos resultados reforçam a importância biológica da RESEX e enfatizam a necessidade de mais inventários e estudos ornitológicos nesta isolada e ainda pouco conhecida região da Amazônia brasileira.

Palavras-chave: Aves. Brasil. Sudeste do Acre. Sudoeste da Amazônia. Reserva Extrativista Chico Mendes.

I South Dakota State University. Geographic Information Science Center of Excellence. Brookings, South Dakota, U.S.A. (luiz.mestre@ gmail.com).

" Museu Paraense Emílio Goeldi. Programa de Pós Graduação em Zoologia. Belém, Pará, Brasil (biogrego@yahoo.com.br).

III South Dakota State University. Geographic Information Science Center of Excellence. Brookings, South Dakota, U.S.A. (mark.cochrane@ sdstate.edu).

Nv Lancaster University. Lancaster Environment Centre. Lancaster, Lancashire, Inglaterra (josbarlow@gmail.com).

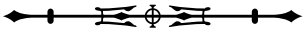




\section{INTRODUCTION}

The western Amazonian bird community is very poorly known, and the Acre avifauna is still being discovered and described. Despite the recent history of ornithological surveys of the State of Acre (less than 60 years of studies), up to 655 bird species have been recorded, and around 75 new species are likely to be included in the State list in the near future (Guilherme, 2009). Given the fast rate of change in our knowledge, all possible information describing the avifauna of this State are significant for the future management and conservation of this important and remote part of the Amazon.

Since the 1950's some researchers have collected and surveyed birds in the State of Acre. One of the first bird collections in this State was led by P. E. Vanzolini in 1951 (Vanzolini, 1952), who confirmed the first 140 bird species for Acre State, and four new species for Brazil (Pinto \& Camargo, 1954). A few other bird surveys took place in Acre in late 1950's and 1960's (Novaes, 1957, 1958), but ornithologists only published the first bird checklists about the avifauna of this region in the 1980's and 1990's (for a detailed description see Guilherme, 2009). Most of the published bird surveys were carried out in the Juruá river Basin in northern Acre (i.e. Novaes, 1957, 1958; Whittaker \& Oren, 1999; Whittaker et al., 2002). Other papers include inventories from the Acre river basin (i.e. Pinto \& Camargo, 1954; Guilherme, 2001). This study describes and highlights the important observations of the avifauna sampled at Reserva Extrativista Chico Mendes (RESEX Chico Mendes), a federal extractive reserve located further south in the Acre river basin, almost at the Brazilian-Peruvian-Bolivian border.

\section{METHODS}

This study was conducted in RESEX Chico Mendes $\left(10^{\circ}\right.$ to $11^{\circ} \mathrm{S}, 68^{\circ}$ to $\left.70^{\circ} \mathrm{W}\right)$, in Acre State, Southwest Amazon, Brazil (Figure 1). This reserve has approximately 1-milion hectares of 'terra firme', non-flooded, openedcanopy forests, with understory dominated by lianas, small palms and/or bamboos (Costa, 2000). The Chico Mendes Reserve is located in a region with annual average temperature around $24^{\circ} \mathrm{C}$, and a mean precipitation of 2,000 $\mathrm{mm}$ (Costa, 2000). The rainy period is from December to March and it is drier from June to August (Costa, 2000). After an extended drought in the region, combined with slash-and-burn techniques used by most of the reserve inhabitants, about 300,000 ha of forest in the reserve were accidentally burned during August and October 2005 (Brown et al., 2006; INPE, 2010). For this reason, the study site is a mosaic of burned and unburned primary Amazonian 'terra firme' forests with patches dominated by bamboos. Most of the burned areas were forested and had been regenerating for three years at the time of the study in 2008. We conducted samples in the end of the dry season, during October $5^{\text {th }}$ and November $10^{\text {th }} 2008$, in the center of the reserve and approximately $100 \mathrm{~km}$ from Bolivian-Peruvian border, in Xapuri municipality (Figure 1). By unburned forests, we refer to sites that had not burned in the 2005 drought but the presence of bamboo in the region may indicate a relatively recent history of fire (Nelson \& Irmão, 1998).

We sampled twelve $550 \mathrm{~m}$ trails that were all located at least $1 \mathrm{~km}$ apart from one another. We used mist-nets, point counts and random observations (between the coordinates $10^{\circ} 13^{\prime} \mathrm{S}, 68^{\circ} 44^{\prime} \mathrm{W}$ and $10^{\circ} 32^{\prime} \mathrm{S}, 68^{\circ} 42^{\prime}$ W) (Figure 1). We performed the random observations at other sites, not just along the trails, including main roads and plantations. For each avifaunal sampling trail, 28 mist-nets $(12 \times 2.5 \mathrm{~m}$; mesh size $36 \mathrm{~mm})$ were erected in four groups of seven nets along $550 \mathrm{~m}$ transects. Each group created a netline of $7 \times 12 \mathrm{~m}$ extending for $90-100 \mathrm{~m}$. Groups were separated by an open space of $50 \mathrm{~m}$. We opened the 28 nets for two days, from sunset $(6: 30 \mathrm{~h})$ to about 13:30 h, accruing 2,050 mist-net hours in total (a capture effort of $11,720 \mathrm{hm}^{2}$ ). We checked the nets hourly and closed it during periods of heavy rain. All birds captured were identified to species level, weighed, and measured (standard measurements included wing, tail, bill, and total length) and, 


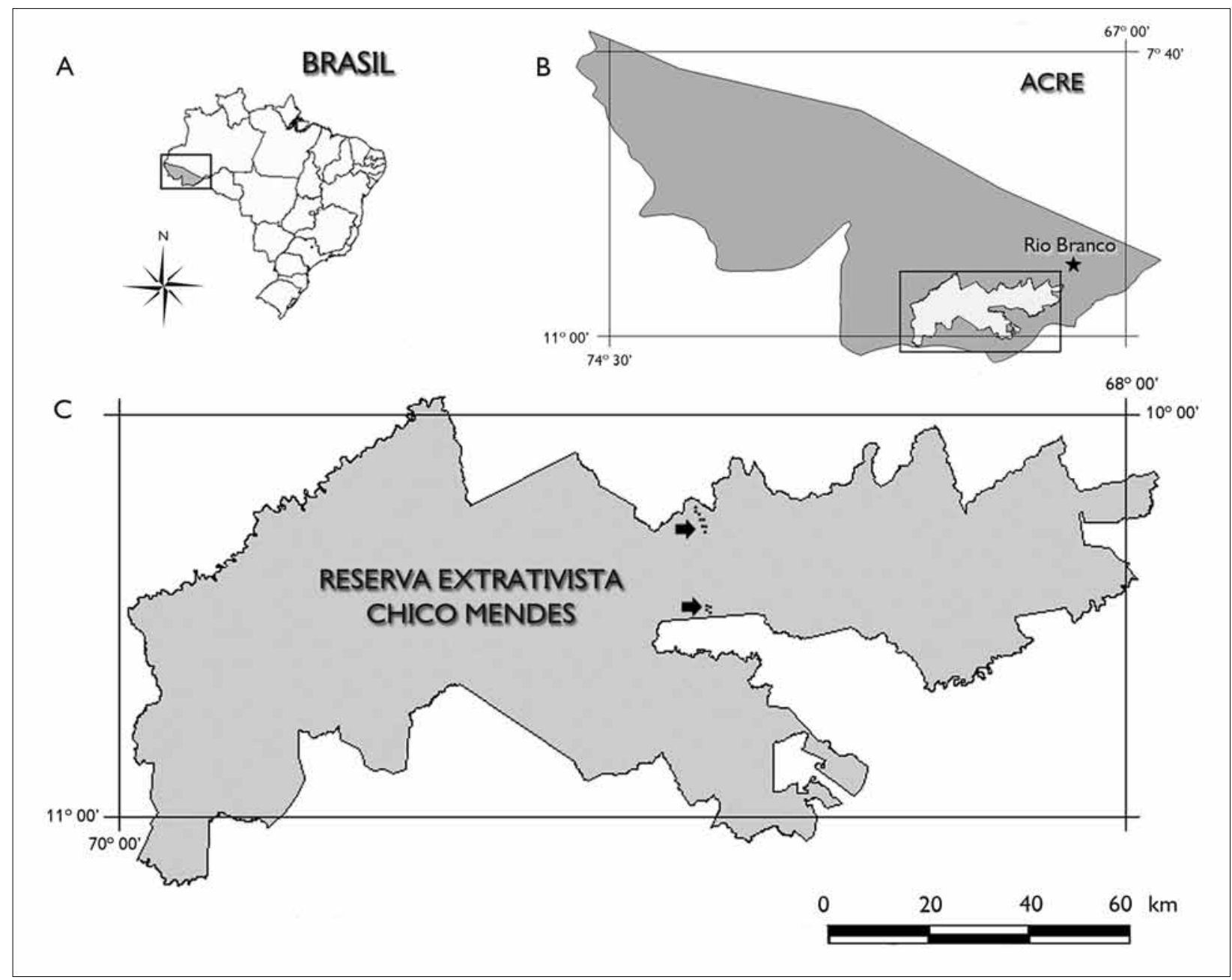

Figure 1. Map of Brazil (A), the State of Acre (B), and RESEX Chico Mendes (C). Arrows and dots showing approximate location of sampling trails (map adapted from Costa, 2000).

whenever possible, were aged, sexed and photographed. The captured birds were banded with a numbered metal ring obtained from Centro Nacional de Pesquisa para Conservação de Aves Silvestres (CEMAVE) - Instituto Chico Mendes de Conservação e Biodiversidade (ICMBio) (The Brazilian Bird Banding Laboratory in the Brazilian National Institute of Environment). All recaptures from the same sampling period and from the same net line were excluded from the analysis to avoid double counting.

In addition to the mist-netting, Luiz Mestre carried out 96 point counts along the trails. The point counts were accomplished two times at the same point on two different days, but not the same days as the mist-netting. First sampling was conducted between 6:30 $\mathrm{h}$ and 7:30 $\mathrm{h}$, and the second between 7:30 h and 9:00 h. Each point count consisted of ten minutes of bird observations and recording, spaced $150 \mathrm{~m}$ each other $(50 \mathrm{~m}, 200 \mathrm{~m}, 350 \mathrm{~m}$ and $500 \mathrm{~m}$ along each transect). All bird registrations were recorded using a digital recorder and a directional microphone during point counts and confirmed (if possible) visually by binoculars. Unknown vocalizations were subsequently checked against known calls and, if necessary, confirmed by consulting with

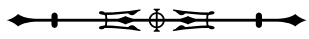


other experienced ornithologists (i.e. A. Aleixo, S. Dantas, E. Guilherme). The distance from the observer and the height at first detection were also noted. We excluded from the richness and abundance analysis all the birds flying or registered outside the range of $50 \mathrm{~m}$ radius of each point count. We also did not include birds that came from directions of other sampling points (primarily wide-ranging canopy species such as Pscitacidae, Embereziidae and Thraupidae), to avoid double counting. Individuals of most species are unlikely to have been registered on consecutive days, because sampling was always carried out at different times of the day. However, we may have inflated numbers of individuals for some territorial species. For this reason, we compared the cumulative curves in three different ways. First, we plotted the curves using only first day sampling separately (Figure 2, PC1). Second, we plotted both days together including all registrations (Figure 2, PC2). Third, we only included the first detection event of a species at a point, using day two only to accumulate species we did not observe on day one. If a species was recorded on the first day and then again on day two, we excluded the day two observation (Figure 2, PC3).

We analyzed the three methods used separately (mist-nets, point counts, general observations) based on number of individuals and percentages. We used percentages of individuals classified separately in the levels of order, family and species. For this reason, the sum of percentages of different categories (taxonomical level) does not reflect the total of upper taxonomical level. We used point counts and mist-net data to calculate rarefaction species curves based upon number of individuals. We calculated these curves with incidence data from point counts and mist-nets separately. We analyzed the patterns of species richness between different methods comparing individual-based rarefaction curves constructed using the analytical formulae from EstimateS v.7 (Colwell, 2004). The comparisons were standardized by of the number of individuals, as we were interested in patterns of species richness and not species density (Gotelli \&
Colwell, 2001). An estimate of the 'true' species richness in each quantitative method (mist-nets and point counts) were calculated using the software EstimateS v.7, using the mean of the four commonly employed abundancebased estimators (ACE, CHAO1, JACK1 [jackknife], and BOOTSTRAPPING). We followed the CBRO (2009) list for nomenclature and taxon ordinance.

\section{RESULTS AND DISCUSSION}

We registered a total of 344 bird species at RESEX Chico Mendes. These bird species were included in 17 orders and 57 families. The most representative families were Tyrannidae, Thamnophilidae and Thraupidae, with 53, 36 and 22 species respectively. Mist-nets captured 868 individuals comprising 137 species from 33 families, and point counts recorded 2,380 individuals from 186 species in 38 families. Mist-nets added 55 species, and general observations added 105 species to the total number of species.

Atthe order level, we captured $77.3 \%$ of Passeriformes in mist-nets. Considering only families, we captured mainly Thamnophilidae (33.5\%), Dendrocolaptidae (12.3\%), Throchilidae (11.2\%) and Tyrannidae (6.6\%). The guilds of captured individuals were represented mainly by arboreal sallying insectivores (20.4\%), arboreal gleaning insectivores (18.8\%), nectarivores (11.2\%), and terrestrial gleaning insectivores (10.5\%). The most captured species were Myrmoborus myotherinus (3.6\%), Arremon taciturnus (3.3\%), Pipra fasciicauda (3.3\%), Phaethornis hispidus (3.2\%), and Myrmoborus leucophrys (3\%). Based on Stotz et al. (1996), roughly $47.4 \%$ of captured birds were medium sensitive species while $44.5 \%$ were highly sensitive to human disturbances. Most of captured individuals (47.8\%) use only one type of habitat. Approximately $42 \%$ of all birds captured by mist-nets use mainly the midstory and understory strata, and $41.6 \%$ use only the understory.

In point counts we detected mostly birds from families Psittacidae (20.8\%), Thamnophilidae (20.7\%), Thraupidae (8.8\%), and Tyrannidae (6.8\%). We registered $20.7 \%$ of arboreal granivorous birds, $17.9 \%$ of arboreal gleaning

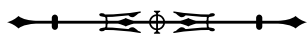




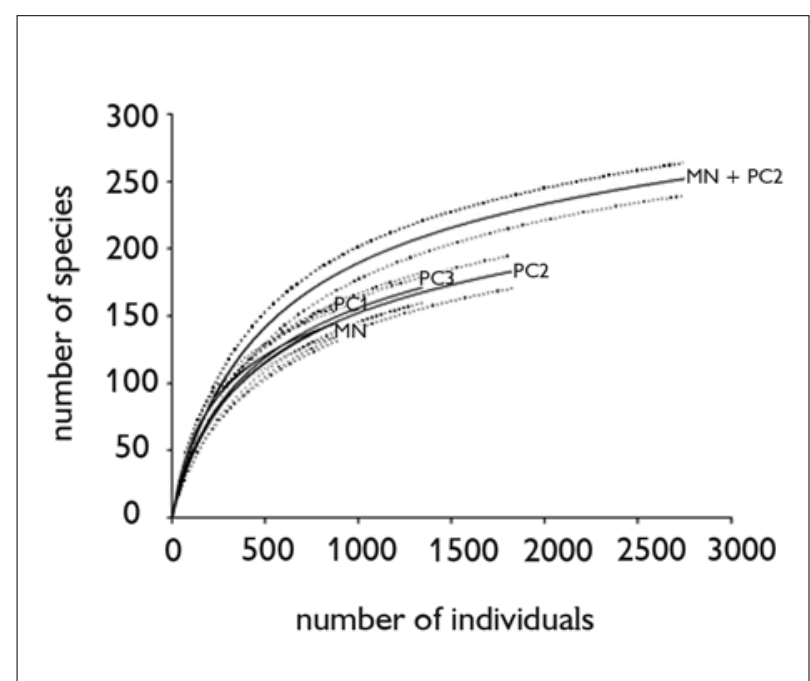

Figure 2. Rarefaction curve from EstimateS Software based on mist-nets ( $M N)$, point-counts (PC1, only first day sampling each trail considered; PC2, all samples considered; PC3 using the second day only to accumulate species not seen in the first day), and the two methods together $(\mathrm{MN}+\mathrm{PC2})$, where estimates are solid lines and 95\% confidence interval dotted line.

insectivores, $16.9 \%$ of arboreal sallying insectivores, and $14.3 \%$ of arboreal omnivores. The most abundant species were Pyrrhura picta (7.1\% of the registrations), Amazona farinosa (4.2\%), Aratinga weddellii (3.3\%), Tangara chilensis (3.1\%), Myrmeciza hemimelaena (2.9\%), and Thamnophilus schistaceus (2.9\%). Forty three percent of these species were classified by Stotz et al. (1996) as highly sensitive species, and $42.1 \%$ as a medium sensitivity bird species. Most of individuals detected by point counts (36.5\%) use only one type of habitat, and $29.9 \%$ use two habitats. About $51.4 \%$ of individuals registered by this method were canopy species.

The rarefaction curves from mist-nets, point-counts or both methods together did not reach an asymptote. However, point counts and both methods summed, showed a possible beginning of stabilization (Figure 2). The point count estimate of the mean 'true' richness was 213.1 ( \pm 13.6 ) species, using the four predicted abundancebased estimators' means (see methods). Mist-net estimated richness was $169.5( \pm 9.3)$ species using the mean of the four estimators. It is possible to observe a better-estimated richness on associating both sampling methods, resulting in 288.3 ( \pm 13.8) species (Table 1). However, it is important to consider that these numbers were from limited samplings based only on point counts and mist-nets and for only one specific season during one year. This result shows the importance of supplementing standardized methods with general observations when a complete species list is the desired outcome. The random observation method added 106 bird species to this checklist, permitting us to estimate for this period about 400 species in the area if combining this method, point count estimate, and mist-net estimate $(106+288.32=394.32)$.

We highlight important observations of restricted range and little known southeast Amazon birds (Table 2). Relevant examples include the following species. Crypturellus atrocapillus, a species limited to the western Amazon (Inambari endemism range). In Brazil, it was only recorded in Acre State; in Parque Nacional da Serra do Divisor (Guilherme, 2009) and Reserva Extrativista do Alto Juruá (Whittaker \& Oren, 1999). We recorded C. atrocapillus mostly in second growth and post-burned sites. Crypturellus strigulosus is a species with a restricted Amazonian range, apparently using mostly Campinas and Campinaranas habitats (Guilherme, 2009). We recorded this rare species in three sampling sites both in pristine and post-burned areas. Jabiru mycteria is included in a secondary list and has few records for Acre State (Guilherme, 2009); we saw a dead juvenile hunted by a local resident. We have no evidence of this registration (picture or skin) but the easy identification of this distinctive species means we were able to include this species in the list. Primolius couloni is a species that is globally vulnerable due to habitat degradation and illegal trade (Birdlife International, 2010; IUCN, 2000); and the limited range in east Peru, north Bolivia and southwest Brazil, also contributes to its vulnerability (Tobias \& Brightsmith, 2007). In Brazil, P. couloni was mainly observed in Acre State (Whittaker \& Oren, 1999; Guilherme, 2009). In the study sites, we observed the species flying and perched in pairs, mostly in pristine forests, and on few occasions, we registered larger 
Table 1. The mean of the 'true' species richness, estimated by point-counts $(P C)$, mist-nets $(M N)$, and both together $(P C+M N)$ by the four richness estimators calculated in EstimateS v.7 software; ACE, Chao, Jack (Jackknife), Bootstrapping, and total mean of the estimators. In parentesis are standard deviations of these means.

\begin{tabular}{lccccc}
\hline & ACE Mean & Chao 1 Mean & Jack 1 Mean & Bootstraping Mean & Total Mean ( \pm SD) \\
\hline PC & $204.95( \pm 0)$ & $207.79( \pm 9.9)$ & $233.47( \pm 6.9)$ & $206.27( \pm 0)$ & $213.12( \pm 13.6)$ \\
MN & $169.72( \pm 0)$ & $162.7( \pm 7.8)$ & $182.59( \pm 6.4)$ & $162.99( \pm 0)$ & $169.5( \pm 9.3)$ \\
$P C+M N$ & $282.21( \pm 0)$ & $283.36( \pm 10.9)$ & $308.79( \pm 7.1)$ & $278.95( \pm 0)$ & $288.3( \pm 13.8)$ \\
\hline
\end{tabular}

groups with about eight individuals. Aulacorhynchus prasinus, despite occurring from Central America to much of western South America, has only been registered a few times in Brazil, and only in Acre State (Sick, 1997; Whittaker \& Oren, 1999). We captured and recorded few individuals of this species in pristine and second growth areas. Percnostola lophotes is a species limited to the western Amazon (Hoyo et al., 2003). In Brazil, it was only recorded in Acre State (Whittaker \& Oren, 1999; Guilherme, 2009). We captured two individuals of $P$. lophotes in pristine and post-burned sites. Hypocnemis peruviana and $H$. subflava are in their range limit and were recorded and photographed in the sites. Their ranges were also confirmed in Guilherme (2009) and Isler et al. (2007). Xiphorhynchus chunchotambo occurs in northwest South America, and in Brazil, this species has only been registered in Acre State (Guilherme, 2009). We registered about ten individuals of this species both in pristine and second growth sites. Conioptilon mcilhennyi occurs in east Peru, north Bolivia and southwest Brazilian Amazonia. This species has only been recorded in Acre State within Brazil, but despite being historically rare, has been associated with second growth and post-burned forests (Guilherme, 2009; Mestre et al., 2009).

Additionally, we underline the importance of this region for the conservation of some North American migratory birds. During this study, we recorded Pandion haliaetus, which uses most of the large Amazonian rivers and breeds in northeastern USA and southern Canada (Hoyo et. al, 1994; Mestre \& Bierregaard, 2009). Tringa solitaria which breeds in north of North America (Alaska, Canada, USA) and migrates to the southern United States, Central and South America (Hoyo et al., 1996). Contopus cooperi that occurs from Alaska (USA) to Southeast Brazil
(Hoyo et al., 2004). Contopus virens, which breeds in eastern North America, migrating to north South America (Sick, 1997; Hoyo et al., 2004). Tyrannus tyrannus occurs from Canada to northern Argentina (Hoyo et al., 2004). Progne subis breeds in North America and north Central America migrating to South America during the Neartic winter (Hoyo et al., 2004). Catharus ustulatus breeds in North America and migrate to South America (Hoyo et al., 2005). Finally, Coccyzus americanus which, despite having Acre within its proposed range (Erize et al., 2006, Restall et al., 2007, Infonatura, 2007), had not previously been registered in the State (Table 2). We clearly observed this species in a forested site, near post-burned forests, perched in the midstory. Although we do not have a documented register of this species, our observation can be considered a first important clue regarding its occurrence in these sites.

The RESEX Chico Mendes forests are also clearly important for avian bamboo specialists, once we recorded 17 species within bamboo formations. Based on Kratter (1997) classification, we found two species that are considered to be obligate bamboo specialists (Drymophila devillei and Hemitriccus flammulatus), seven species of near obligate specialists (Celeus spectabilis, Simoxenops ucayalae, Automolus melanopezus, Percnostola lophotes, Myrmeciza goeldii, Ramphotrigon megacephalum and Ramphotrigon fuscicauda), and three facultative bamboo specialists (Campylorhamphus trochilirostris, Epinecrophylla ornata, and Microrhopias quixensis). We found D. devillei, $H$. flammulatus and $P$. lophotes only in sites with abundant bamboo formations, including some second growth and post-burned areas. For this reason, our data also confirm the restriction of these species to bamboo forests as

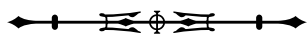


Table 2. Bird species list of RESEX Chico Mendes, South Acre, Brazil. The list follows the CBRO bird checklist from 2009 and specify the taxon (including bird species, family and order); Portuguese and English names of each species; the status (St) of the bird species as resident (R), North migrant $(\mathrm{VN})$ and if the species was registered in point counts (PC), mist-nets (MN) or by general observations (GO) (\# status not confirmed $\mathrm{CBRO}$ ). Habitats where species were registered 1: Primary Forests; 2: Second growth sites including post burned; 3: Plantations, open and/or aquatic habitats; 4: Flying. CA, Categories of Abundance in the sampling sites (c: common, u: uncommon, r: rare); TE, Types of Evidence (r: recording, p: picture, c: collected and deposited in Museu Paraense Emílio Goeldi, o: only observed).

(Continued)

\begin{tabular}{|c|c|c|c|c|c|c|c|c|}
\hline Taxon & Portuguese name & English name & St & PC & $M N$ & GO & CA & TE \\
\hline \multicolumn{9}{|l|}{ Tinamiformes Huxley, 1872} \\
\hline \multicolumn{9}{|l|}{ Tinamidae Gray, 1840} \\
\hline Tinamus tao Temminck, 1815 & Azulona & Gray Tinamou & $\mathrm{R}$ & 1 & & & c & r \\
\hline Tinamus guttatus Pelzeln, 1863 & Inhambu-galinha & $\begin{array}{c}\text { White-throated } \\
\text { Tinamou }\end{array}$ & $\mathrm{R}$ & 1 & 1 & & u & r \\
\hline Crypturellus cinereus (Gmelin, 1789) & Inhambu-preto & $\begin{array}{l}\text { Cinereous } \\
\text { Tinamou }\end{array}$ & $\mathrm{R}$ & 1,2 & 2 & & u & r \\
\hline Crypturellus soui (Hermann, 1783) & Tururim & Little Tinamou & $\mathrm{R}$ & 2,3 & & & c & r \\
\hline Crypturellus obsoletus (Temminck, 1815) & Inhambuguaçu & Brown Tinamou & $\mathrm{R}$ & 1,2 & & & $\mathrm{u}$ & r \\
\hline Crypturellus undulatus (Temminck, 1815) & Jaó & Undulated Tinamou & $\mathrm{R}$ & 1,2 & & & c & r \\
\hline Crypturellus strigulosus (Temminck, 1815) & Inhambu-relógio & Brazilian Tinamou & $\mathrm{R}$ & 1,2 & & & r & r \\
\hline Crypturellus atrocapillus (Tschudi, 1844) & Inhambu-de-coroa-preta & Black-capped Tinamou & $\mathrm{R}$ & 1,2 & & & u & r \\
\hline Crypturellus variegatus (Gmelin, 1789) & Inhambu-anhangá & Variegated Tinamou & $\mathrm{R}$ & & & 1,2 & & \\
\hline \multicolumn{9}{|l|}{ Anseriformes Linnaeus, 1758} \\
\hline \multicolumn{9}{|l|}{ Anatidae Leach, 1820} \\
\hline Amazonetta brasiliensis (Gmelin, 1789) & Pé-vermelho & Brazilian Teal & $\mathrm{R}$ & & & 3 & u & O \\
\hline \multicolumn{9}{|l|}{ Galliformes Linnaeus, 1758} \\
\hline \multicolumn{9}{|l|}{ Cracidae Rafinesque, 1815} \\
\hline Ortalis guttata (Spix, 1825) & Aracuã & Speckled Chachalaca & $\mathrm{R}$ & 1,2 & & & c & r \\
\hline Penelope jacquacu Spix, 1825 & Jacu-de-spix & Spix's Guan & $\mathrm{R}$ & 1,2 & & & r & O \\
\hline Aburria cujubi (Pelzeln, 1858) & Cujubi & $\begin{array}{l}\text { Red-throated } \\
\text { Piping Guan }\end{array}$ & $\mathrm{R}$ & & & 1 & r & O \\
\hline \multicolumn{9}{|l|}{ Odontophoridae Gould, 1844} \\
\hline Odontophorus stellatus (Gould, 1843) & Uru-de-topete & Starred Wood-Quail & $\mathrm{R}$ & 1,2 & & & r & r \\
\hline \multicolumn{9}{|l|}{ Ciconiiformes Bonaparte, 1854} \\
\hline \multicolumn{9}{|l|}{ Ardeidae Leach, 1820} \\
\hline Tigrisoma lineatum (Boddaert, 1783) & Socó-boi & Rufescent Tiger-Heron & $\mathrm{R}$ & & & 3 & r & $\mathrm{O}$ \\
\hline Butorides striata (Linnaeus, 1758) & Socozinho & Striated Heron & $\mathrm{R}$ & & & 3 & u & $\mathrm{O}$ \\
\hline Pilherodius pileatus (Boddaert, 1783) & Garça-real & Capped Heron & $\mathrm{R}$ & & & 3 & u & $\mathrm{O}$ \\
\hline \multicolumn{9}{|l|}{ Ciconiidae Sundevall, 1836} \\
\hline Jabiru mycteria (Lichtenstein, 1819) & Tuiuiú & Jabiru & $\mathrm{R}$ & & & 3 & r & $\mathrm{O}$ \\
\hline \multicolumn{9}{|l|}{ Cathartiformes Seebohm, 1890} \\
\hline Cathartidae Lafresnaye, 1839 & & & & & & & & \\
\hline
\end{tabular}


Table 2.

(Continued)

\begin{tabular}{|c|c|c|c|c|c|c|c|c|}
\hline Taxon & Portuguese name & English name & St & PC & $M N$ & GO & CA & TE \\
\hline Cathartes aura (Linnaeus, 1758) & Urubu-de-cabeça-vermelha & Turkey Vulture & $\mathrm{R}$ & & & 4 & $c$ & o \\
\hline Cathartes melambrotus Wetmore, 1964 & Urubu-da-mata & $\begin{array}{c}\text { Greater Yellow-headed } \\
\text { Vulture }\end{array}$ & $\mathrm{R}$ & & & 4 & u & O \\
\hline Coragyps atratus (Bechstein, 1793) & Urubu-de-cabeça-preta & Black Vulture & $\mathrm{R}$ & & & 4 & c & O \\
\hline Sarcoramphus papa (Linnaeus, 1758) & Urubu-rei & King Vulture & $\mathrm{R}$ & & & 4 & r & o \\
\hline \multicolumn{9}{|l|}{ Falconiformes Bonaparte, 1831} \\
\hline \multicolumn{9}{|l|}{ Pandionidae Bonaparte, 1854} \\
\hline Pandion haliaetus (Linnaeus, 1758) & Águia-pescadora & Osprey & VN & & & 4 & r & o \\
\hline \multicolumn{9}{|l|}{ Accipitridae Vigors, 1824} \\
\hline Elanoides forficatus (Linnaeus, 1758) & Gavião-tesoura & Swallow-tailed Kite & $\mathrm{R}$ & & & 4 & u & O \\
\hline Harpagus bidentatus (Latham, 1790) & Gavião-ripina & Double-toothed Kite & $\mathrm{R}$ & & & 1 & r & O \\
\hline Ictinia plumbea (Gmelin, 1788) & Sovi & Plumbeous Kite & $\mathrm{R}$ & 2,3 & & & c & o \\
\hline Geranospiza caerulescens (Vieillot, 1817) & Gavião-pernilongo & Crane Hawk & $\mathrm{R}$ & & & 4 & r & O \\
\hline Leucopternis albicollis (Latham, 1790) & Gavião-branco & White Hawk & $\mathrm{R}$ & & & 4 & u & o \\
\hline Buteogallus urubitinga (Gmelin, 1788) & Gavião-preto & Great Black-Hawk & $\mathrm{R}$ & & & 4 & r & O \\
\hline Rupornis magnirostris (Gmelin, 1788) & Gavião-carijó & Roadside Hawk & $\mathrm{R}$ & 2,3 & & & $c$ & r \\
\hline Buteo nitidus (Latham, 1790) & Gavião-pedrês & Gray Hawk & $\mathrm{R}$ & & & 2,3 & u & O \\
\hline Spizaetus tyrannus (Wied, 1820) & Gavião-pega-macaco & Black Hawk-Eagle & $\mathrm{R}$ & 1 & & & r & r \\
\hline Spizaetus ornatus (Daudin, 1800) & Gavião-de-penacho & Ornate Hawk-Eagle & $\mathrm{R}$ & 4 & & & r & O \\
\hline \multicolumn{9}{|l|}{ Falconidae Leach, 1820} \\
\hline Daptrius ater Vieillot, 1816 & Gavião-de-anta & Black Caracara & $\mathrm{R}$ & & & 2,3 & c & r \\
\hline Ibycter americanus (Boddaert, 1783) & Gralhão & Red-throated Caracara & $\mathrm{R}$ & 1,2 & & & c & r \\
\hline Caracara plancus (Miller, 1777) & Caracará & Southern Caracara & $\mathrm{R}$ & & & 2,3 & c & r \\
\hline Milvago chimachima (Vieillot, 1816) & Carrapateiro & $\begin{array}{l}\text { Yellow-headed } \\
\text { Caracara }\end{array}$ & $\mathrm{R}$ & & & 2,3 & c & r \\
\hline Herpetotheres cachinnans (Linnaeus, 1758) & Acauã & Laughing Falcon & $\mathrm{R}$ & & & 2,3 & c & r \\
\hline Micrastur ruficollis (Vieillot, 1817) & Falcão-caburé & Barred Forest-Falcon & $\mathrm{R}$ & 1,2 & & & u & r \\
\hline Micrastur gilvicollis (Vieillot, 1817) & Falcão-mateiro & Lined Forest-Falcon & $\mathrm{R}$ & 1,2 & 1,2 & & u & r \\
\hline Micrastur semitorquatus (Vieillot, 1817) & Falcão-relógio & Collared Forest-Falcon & $\mathrm{R}$ & 1,2 & & & u & r \\
\hline Falco rufigularis Daudin, 1800 & Cauré & Bat Falcon & $\mathrm{R}$ & & & 1,2 & u & ○ \\
\hline \multicolumn{9}{|l|}{ Psophiidae Bonaparte, 1831} \\
\hline Psophia leucoptera Spix, 1825 & Jacamim-de-costas-brancas & Pale-winged Trumpeter & $\mathrm{R}$ & & & 1,2 & u & O \\
\hline \multicolumn{9}{|l|}{ Rallidae Rafinesque, 1815} \\
\hline Aramides cajanea (Statius Muller, 1776) & Saracura-três-potes & $\begin{array}{l}\text { Gray-necked } \\
\text { Wood-Rail }\end{array}$ & $\mathrm{R}$ & & & 2,3 & c & r \\
\hline Anurolimnas castaneiceps (Sclater \& Salvin, 1869) & Sanã-de-cabeça-castanha & Chestnut-headed Crake & R\# & 2,3 & & & r & r \\
\hline Heliornithidae Gray, 1840 & & & & & & & & \\
\hline
\end{tabular}

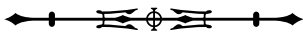


Table 2.

(Continued)

\begin{tabular}{|c|c|c|c|c|c|c|c|c|}
\hline Taxon & Portuguese name & English name & St & PC & $M N$ & GO & CA & TE \\
\hline Heliornis fulica (Boddaert, 1783) & Picaparra & Sungrebe & $\mathrm{R}$ & & & 2,3 & r & ○ \\
\hline \multicolumn{9}{|l|}{ Eurypygidae Selby, 1840} \\
\hline Eurypyga helias (Pallas, 1781) & Pavãozinho-do-pará & Sunbittern & $\mathrm{R}$ & & & 1,2 & r & O \\
\hline \multicolumn{9}{|l|}{ Charadriiformes Huxley, 1867} \\
\hline \multicolumn{9}{|l|}{ Charadriidae Leach, 1820} \\
\hline Vanellus chilensis (Molina, 1782) & Quero-quero & Southern Lapwing & $\mathrm{R}$ & & & 3 & $c$ & r \\
\hline \multicolumn{9}{|l|}{ Scolopacidae Rafinesque, 1815} \\
\hline Tringa solitaria Wilson, 1813 & Maçarico-solitário & Solitary Sandpiper & VN & & & 3 & r & o \\
\hline \multicolumn{9}{|l|}{ Jacanidae Chenu \& Des Murs, 1854} \\
\hline Jacana jacana (Linnaeus, 1766) & Jaçanã & Wattled Jacana & $\mathrm{R}$ & & & 3 & c & O \\
\hline \multicolumn{9}{|l|}{ Columbidae Leach, 1820} \\
\hline Columbina talpacoti (Temminck, 1811) & Rolinha-roxa & Ruddy Ground-Dove & $\mathrm{R}$ & & & 2,3 & $c$ & r \\
\hline Claravis pretiosa (Ferrari-Perez, 1886) & Pararu-azul & Blue Ground-Dove & $\mathrm{R}$ & & & 1,2 & c & r \\
\hline Patagioenas plumbea (Vieillot, 1818) & Pomba-amargosa & Plumbeous Pigeon & $\mathrm{R}$ & 1,2 & & & c & r \\
\hline Patagioenas subvinacea (Lawrence, 1868) & Pomba-botafogo & Ruddy Pigeon & $\mathrm{R}$ & 1,2 & & & c & r \\
\hline Leptotila rufaxilla (Richard \& Bernard, 1792) & Juriti-gemedeira & Gray-fronted Dove & $\mathrm{R}$ & 1,2 & 2 & & c & r \\
\hline Geotrygon montana (Linnaeus, 1758) & Pariri & Ruddy Quail-Dove & $\mathrm{R}$ & 1 & 1 & & c & $p$ \\
\hline \multicolumn{9}{|l|}{ Psittaciformes Wagler, 1830} \\
\hline \multicolumn{9}{|l|}{ Psittacidae Rafinesque, 1815} \\
\hline Ara macao (Linnaeus, 1758) & Araracanga & Scarlet Macaw & $\mathrm{R}$ & 1,2 & & & u & r \\
\hline Ara chloropterus Gray, 1859 & Arara-vermelha-grande & Red-and-green Macaw & $\mathrm{R}$ & 1,2 & & & u & r \\
\hline Ara severus (Linnaeus, 1758) & Maracanã-guaçu & $\begin{array}{c}\text { Chestnut-fronted } \\
\text { Macaw }\end{array}$ & $\mathrm{R}$ & 1,2 & & & u & r \\
\hline Primolius couloni (Sclater, 1876) & Maracanã-de-cabeça-azul & Blue-headed Macaw & $R$ & 1,2 & & & u & r \\
\hline Aratinga leucophthalma (Statius Muller, 1776) & Periquitão-maracanã & White-eyed Parakeet & $\mathrm{R}$ & 1,2 & & & c & r \\
\hline Aratinga weddellii (Deville, 1851) & Periquito-de-cabeça-suja & Dusky-headed Parakeet & $\mathrm{R}$ & 1,2 & & & c & r \\
\hline Pyrrhura roseifrons (Gray, 1859) & Tiriba-de-cabeça-vermelha & Red-crowned Parakeet & $\mathrm{R}$ & 1,2 & & & c & r \\
\hline Pyrrhura rupicola (Tschudi, 1844) & Tiriba-rupestre & Black-capped Parakeet & R\# & & & 1,2 & u & $\mathrm{O}$ \\
\hline Brotogeris cyanoptera (Pelzeln, 1870) & Periquito-de-asa-azul & $\begin{array}{l}\text { Cobalt-winged } \\
\text { Parakeet }\end{array}$ & $\mathrm{R}$ & 1,2 & & & c & $r$ \\
\hline Pionites leucogaster (Kuhl, 1820) & Marianinha-de-cabeça-amarela & White-bellied Parrot & $\mathrm{R}$ & 1,2 & & & c & r \\
\hline Pionus menstruus (Linnaeus, 1766) & Maitaca-de-cabeça-azul & Blue-headed Parrot & $\mathrm{R}$ & 1,2 & & & c & r \\
\hline Amazona farinosa (Boddaert, 1783) & Papagaio-moleiro & Mealy Parrot & $\mathrm{R}$ & 1,2 & & & c & r \\
\hline Amazona ochrocephala (Gmelin, 1788) & Papagaio-campeiro & Yellow-crowned Parrot & $\mathrm{R}$ & 1,2 & & & $r$ & $r$ \\
\hline Cuculiformes Wagler, 1830 & & & & & & & & \\
\hline
\end{tabular}


Table 2.

(Continued)

\begin{tabular}{|c|c|c|c|c|c|c|c|c|}
\hline Taxon & Portuguese name & English name & St & PC & $\mathrm{MN}$ & GO & CA & TE \\
\hline \multicolumn{9}{|l|}{ Cuculidae Leach, 1820} \\
\hline Piaya cayana (Linnaeus, 1766) & Alma-de-gato & Squirrel Cuckoo & $\mathrm{R}$ & 1,2 & & & C & r \\
\hline Piaya melanogaster (Vieillot, 1817) & Chincoã-de-bico-vermelho & Black-bellied Cuckoo & $\mathrm{R}$ & 1,2 & & & u & r \\
\hline Coccyzus americanus (Linnaeus, 1758) & Papa-lagarta-de-asa-vermelha & Yellow-billed Cuckoo & VN & 1,2 & & & r & o \\
\hline Crotophaga major Gmelin, 1788 & Anu-coroca & Greater Ani & $\mathrm{R}$ & & & 2,3 & $c$ & o \\
\hline Crotophaga ani Linnaeus, 1758 & Anu-preto & Smooth-billed Ani & $\mathrm{R}$ & & & 2,3 & c & r \\
\hline Tapera naevia (Linnaeus, 1766) & Saci & Striped Cuckoo & $\mathrm{R}$ & & & 2 & $\mathrm{u}$ & ० \\
\hline \multicolumn{9}{|l|}{ Strigiformes Wagler, 1830} \\
\hline \multicolumn{9}{|l|}{ Tytonidae Mathews, 1912} \\
\hline Tyto alba (Scopoli, 1769) & Coruja-da-igreja & Barn Owl & $\mathrm{R}$ & & & 3 & $\mathrm{u}$ & O \\
\hline \multicolumn{9}{|l|}{ Strigidae Leach, 1820} \\
\hline Megascops choliba (Vieillot, 1817) & Corujinha-do-mato & Tropical Screech-Owl & $\mathrm{R}$ & & & 2,3 & $\mathrm{c}$ & r \\
\hline Megascops usta (Sclater, 1858)) & Corujinha-orelhuda & $\begin{array}{l}\text { Tawny-bellied } \\
\text { Screech-Owl }\end{array}$ & $\mathrm{R}$ & & & 1,2 & C & r \\
\hline Pulsatrix perspicillata (Latham, 1790) & Murucututu & Spectacled Owl & $\mathrm{R}$ & & & 1,2 & r & ० \\
\hline Glaucidium hardyi Vielliard, 1990 & Caburé-da-amazônia & Amazonian Pygmy-Owl & $\mathrm{R}$ & 1,2 & & & $\mathrm{u}$ & r \\
\hline Glaucidium brasilianum (Gmelin, 1788) & Caburé & Ferruginous Pygmy-Owl & $\mathrm{R}$ & 1 & & & u & r \\
\hline Athene cunicularia (Molina, 1782) & Coruja-buraqueira & Burrowing Owl & $\mathrm{R}$ & & & 3 & C & r \\
\hline \multicolumn{9}{|l|}{ Caprimulgiformes Ridgway, 1881} \\
\hline \multicolumn{9}{|l|}{ Nyctibiidae Chenu \& Des Murs, 1851} \\
\hline Nyctibius grandis (Gmelin, 1789) & Mãe-da-lua-gigante & Great Potoo & $\mathrm{R}$ & & & 1,2 & r & r \\
\hline Nyctibius griseus (Gmelin, 1789) & Mãe-da-lua & Common Potoo & $\mathrm{R}$ & & & 1,2 & $\mathrm{u}$ & r \\
\hline \multicolumn{9}{|l|}{ Caprimulgidae Vigors, 1825} \\
\hline Nyctidromus albicollis (Gmelin, 1789) & Bacurau & Pauraque & $\mathrm{R}$ & & & 2,3 & c & r \\
\hline \multicolumn{9}{|l|}{ Apodiformes Peters, 1940} \\
\hline \multicolumn{9}{|l|}{ Apodidae Olphe-Galliard, 1887} \\
\hline Chaetura egregia Todd, 1916 & Taperá-de-garganta-branca & Pale-rumped Swift & $\mathrm{R}$ & 4 & & & r & r \\
\hline Chaetura brachyura (Jardine, 1846) & Andorinhão-de-rabo-curto & Short-tailed Swift & $\mathrm{R}$ & & & 4 & $\mathrm{u}$ & o \\
\hline \multicolumn{9}{|l|}{ Trochilidae Vigors, 1825} \\
\hline Glaucis hirsutus (Gmelin, 1788) & Balança-rabo-de-bico-torto & $\begin{array}{c}\text { Rufous-breasted } \\
\text { Hermit }\end{array}$ & $\mathrm{R}$ & 1,2 & 1,2 & & u & $\mathrm{P}$ \\
\hline Threnetes leucurus (Linnaeus, 1766) & $\begin{array}{c}\text { Balança-rabo-de- } \\
\text { garganta-preta }\end{array}$ & Pale-tailed Barbthroat & $\mathrm{R}$ & & 1,2 & & u & $\mathrm{p}$ \\
\hline Phaethornis ruber (Linnaeus, 1758) & Rabo-branco-rubro & Reddish Hermit & $\mathrm{R}$ & & & 1,2 & $\mathrm{C}$ & $\mathrm{p}$ \\
\hline Phaethornis hispidus (Gould, 1846) & Rabo-branco-cinza & White-bearded Hermit & $\mathrm{R}$ & & 1,2 & & u & $\mathrm{p}$ \\
\hline Phaethornis philippii (Bourcier, 1847) & Rabo-branco-amarelo & Needle-billed Hermit & $\mathrm{R}$ & & 1,2 & & r & $\mathrm{p}$ \\
\hline Phaethornis malaris (Nordmann, 1835) & Besourão-de-bico-grande & Great-billed Hermit & $\mathrm{R}$ & 1,2 & 1,2 & & r & $p$ \\
\hline
\end{tabular}

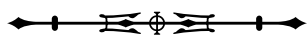


Table 2.

(Continued)

\begin{tabular}{|c|c|c|c|c|c|c|c|c|}
\hline Taxon & Portuguese name & English name & St & PC & $M N$ & GO & CA & TE \\
\hline Anthracothorax nigricollis (Vieillot, 1817) & Beija-flor-de-veste-preta & Black-throated Mango & $\mathrm{R}$ & 2 & & & r & ० \\
\hline Thalurania furcata (Gmelin, 1788) & Beija-flor-tesoura-verde & $\begin{array}{l}\text { Fork-tailed } \\
\text { Woodnymph }\end{array}$ & $\mathrm{R}$ & 1,2 & 1,2 & & u & $\mathrm{P}$ \\
\hline Hylocharis cyanus (Vieillot, 1818) & Beija-flor-roxo & $\begin{array}{l}\text { White-chinned } \\
\text { Sapphire }\end{array}$ & $\mathrm{R}$ & 1,2 & 1,2 & & u & $P$ \\
\hline Amazilia lactea (Lesson, 1832) & Beija-flor-de-peito-azul & $\begin{array}{c}\text { Sapphire-spangled } \\
\text { Emerald }\end{array}$ & $\mathrm{R}$ & & 1,2 & & r & $p$ \\
\hline Heliothryx auritus (Gmelin, 1788) & Beija-flor-de-bochecha-azul & Black-eared Fairy & $\mathrm{R}$ & & 1 & & r & $\mathrm{p}$ \\
\hline \multicolumn{9}{|l|}{ Trogoniformes A. O. U., 1886} \\
\hline \multicolumn{9}{|l|}{ Trogonidae Lesson, 1828} \\
\hline Trogon melanurus Swainson, 1838 & Surucuá-de-cauda-preta & Black-tailed Trogon & $\mathrm{R}$ & 1,2 & & & c & r \\
\hline Trogon viridis Linnaeus, 1766 & $\begin{array}{l}\text { Surucuá-grande-de- } \\
\text { barriga-amarela }\end{array}$ & White-tailed Trogon & $\mathrm{R}$ & 1,2 & & & u & r \\
\hline Trogon rufus Gmelin, 1788 & Surucuá-de-barriga-amarela & Black-throated Trogon & $\mathrm{R}$ & 1 & & & r & r \\
\hline Trogon collaris Vieillot, 1817 & Surucuá-de-coleira & Collared Trogon & $\mathrm{R}$ & 1,2 & 1,2 & & u & r \\
\hline \multicolumn{9}{|l|}{ Coraciiformes Forbes, 1844} \\
\hline \multicolumn{9}{|l|}{ Alcedinidae Rafinesque, 1815} \\
\hline Chloroceryle amazona (Latham, 1790) & Martim-pescador-verde & Amazon Kingfisher & $\mathrm{R}$ & & & 3 & u & O \\
\hline Chloroceryle aenea (Pallas, 1764) & Martinho & $\begin{array}{l}\text { American Pygmy } \\
\text { Kingfisher }\end{array}$ & $\mathrm{R}$ & & 1,2 & & r & $P$ \\
\hline Chloroceryle inda (Linnaeus, 1766) & Martim-pescador-da-mata & $\begin{array}{l}\text { Green-and-rufous } \\
\text { Kingfisher }\end{array}$ & $\mathrm{R}$ & & 1,2 & & r & $P$ \\
\hline \multicolumn{9}{|l|}{ Momotidae Gray, 1840} \\
\hline Electron platyrhynchum (Leadbeater, 1829) & Udu-de-bico-largo & Broad-billed Motmot & $\mathrm{R}$ & 1,2 & & & r & r \\
\hline Baryphthengus martii (Spix, 1824) & Juruva-ruiva & Rufous Motmot & $\mathrm{R}$ & 1,2 & 1,2 & & r & $\mathrm{p}$ \\
\hline Momotus momota (Linnaeus, 1766) & Udu-de-coroa-azul & Blue-crowned Motmot & $\mathrm{R}$ & 1,2 & 1,2 & & c & $r, p$ \\
\hline \multicolumn{9}{|l|}{ Galbuliformes Fürbringer, 1888} \\
\hline \multicolumn{9}{|l|}{ Galbulidae Vigors, 1825} \\
\hline Galbula cyanescens Deville, 1849 & Ariramba-da-capoeira & Bluish-fronted Jacamar & $\mathrm{R}$ & 1,2 & 1,2 & & c & $r, p$ \\
\hline Galbula dea (Linnaeus, 1758) & Ariramba-do-paraíso & Paradise Jacamar & $\mathrm{R}$ & 1,2 & & & c & r \\
\hline Jacamerops aureus (Statius Muller, 1776) & Jacamaraçu & Great Jacamar & $R$ & & & 1,2 & u & r \\
\hline \multicolumn{9}{|l|}{ Bucconidae Horsfield, 1821} \\
\hline Notharchus hyperrhynchus (Sclater, 1856) & Macuru-de-testa-branca & White-necked Puffbird & $\mathrm{R}$ & 1,2 & & & u & r \\
\hline Nystalus striolatus (Pelzeln, 1856) & Rapazinho-estriado & Striolated Puffbird & $\mathrm{R}$ & & & 1,2 & u & O \\
\hline Malacoptila semicincta Todd, 1925 & Barbudo-de-coleira & Semicollared Puffbird & $\mathrm{R}$ & 1,2 & 1,2 & & u & $r, p$ \\
\hline Nonnula ruficapilla (Tschudi, 1844) & Freirinha-de-coroa-castanha & Rufous-capped Nunlet & $\mathrm{R}$ & & 1,2 & & u & $\mathrm{p}$ \\
\hline Monasa nigrifrons (Spix, 1824) & Chora-chuva-preto & Black-fronted Nunbird & $\mathrm{R}$ & 1,2 & 1,2 & & u & $r, p$ \\
\hline
\end{tabular}


Table 2.

(Continued)

\begin{tabular}{|c|c|c|c|c|c|c|c|c|}
\hline Taxon & Portuguese name & English name & St & PC & $M N$ & GO & CA & TE \\
\hline Monasa morphoeus (Hahn \& Küster, 1823) & Chora-chuva-de-cara-branca & White-fronted Nunbird & $\mathrm{R}$ & 1,2 & 1,2 & & c & $r, p$ \\
\hline Monasa flavirostris Strickland, 1850 & $\begin{array}{l}\text { Chora-chuva-de- } \\
\text { bico-amarelo }\end{array}$ & Yellow-billed Nunbird & $\mathrm{R}$ & & 1,2 & & r & $\mathrm{P}$ \\
\hline \multicolumn{9}{|l|}{ Piciformes Meyer \& Wolf, 1810} \\
\hline \multicolumn{9}{|l|}{ Capitonidae Bonaparte, 1838} \\
\hline Capito auratus (Dumont, 1816) & Capitão-de-fronte-dourada & Guilded Barbet & $\mathrm{R}$ & 1,2 & & & u & r \\
\hline Eubucco richardsoni (Gray, 1846) & Capitão-de-bigode-limão & Lemon-throated Barbet & $\mathrm{R}$ & 1,2 & & & r & r \\
\hline \multicolumn{9}{|l|}{ Ramphastidae Vigors, 1825} \\
\hline Ramphastos tucanus Linnaeus, 1758 & $\begin{array}{l}\text { Tucano-grande-de- } \\
\text { papo-branco }\end{array}$ & Red-billed Toucan & $\mathrm{R}$ & 1,2 & & & c & r \\
\hline Ramphastos vitellinus Lichtenstein, 1823 & Tucano-de-bico-preto & Channel-billed Toucan & $\mathrm{R}$ & 1,2 & 1,2 & & c & $r, p$ \\
\hline Aulacorhynchus prasinus & Tucano-verde & Emerald Toucanet & $\mathrm{R}$ & 1,2 & 1,2 & & r & $p, c$ \\
\hline Selenidera reinwardtii (Wagler, 1827) & Saripoca-de-coleira & $\begin{array}{l}\text { Golden-collared } \\
\text { Toucanet }\end{array}$ & $\mathrm{R}$ & 1,2 & 1,2 & & r & $\mathrm{p}$ \\
\hline Pteroglossus inscriptus Swainson, 1822 & $\begin{array}{l}\text { Araçari-miudinho-de- } \\
\text { bico-riscado }\end{array}$ & Lettered Aracari & $\mathrm{R}$ & 1,2 & 1,2 & & u & $\mathrm{p}$ \\
\hline Pteroglossus mariae Gould, 1854 & Araçari-de-bico-marrom & $\begin{array}{l}\text { Brown-mandibled } \\
\text { Aracari }\end{array}$ & $\mathrm{R}$ & & 1 & & r & $\mathrm{p}$ \\
\hline Pteroglossus castanotis Gould, 1834 & Araçari-castanho & Chestnut-eared Aracari & $\mathrm{R}$ & 1 & & & u & r \\
\hline Pteroglossus beauharnaesii Wagler, 1832 & Araçari-mulato & Curl-crested Aracari & $\mathrm{R}$ & 1,2 & 1,2 & & r & $\mathrm{p}$ \\
\hline \multicolumn{9}{|l|}{ Picidae Leach, 1820} \\
\hline Melanerpes cruentatus (Boddaert, 1783) & Benedito-de-testa-vermelha & $\begin{array}{l}\text { Yellow-tufted } \\
\text { Woodpecker }\end{array}$ & $\mathrm{R}$ & 1,2 & & & c & r \\
\hline Veniliornis affinis (Swainson, 1821) & Picapauzinho-avermelhado & $\begin{array}{l}\text { Red-stained } \\
\text { Woodpecker }\end{array}$ & $\mathrm{R}$ & & 1 & & u & $\mathrm{p}$ \\
\hline Veniliornis passerinus (Linnaeus, 1766) & Picapauzinho-anão & Little Woodpecker & $\mathrm{R}$ & 1,2 & & & u & $\mathrm{p}$ \\
\hline Piculus flavigula (Boddaert, 1783) & Pica-pau-bufador & $\begin{array}{l}\text { Yellow-throated } \\
\text { Woodpecker }\end{array}$ & $\mathrm{R}$ & 1,2 & & & u & r \\
\hline Piculus chrysochloros (Vieillot, 1818) & Pica-pau-dourado-escuro & $\begin{array}{l}\text { Golden-green } \\
\text { Woodpecker }\end{array}$ & $\mathrm{R}$ & & & 1,2 & u & o \\
\hline $\begin{array}{l}\text { Celeus grammicus } \\
\text { (Natterer \& Malherbe, 1845) }\end{array}$ & Picapauzinho-chocolate & $\begin{array}{l}\text { Scaly-breasted } \\
\text { Woodpecker }\end{array}$ & $\mathrm{R}$ & & & 1,2 & r & o \\
\hline Celeus flavus (Statius Muller, 1776) & Pica-pau-amarelo & $\begin{array}{l}\text { Cream-colored } \\
\text { Woodpecker }\end{array}$ & $\mathrm{R}$ & 1 & & & r & r \\
\hline Celeus spectabilis Sclater \& Salvin, 1880 & Pica-pau-lindo & $\begin{array}{l}\text { Rufous-headed } \\
\text { Woodpecker }\end{array}$ & R\# & & 1,2 & & r & $\mathrm{P}$ \\
\hline Celeus torquatus (Boddaert, 1783) & Pica-pau-de-coleira & Ringed Woodpecker & $\mathrm{R}$ & 1,2 & & & u & $r$ \\
\hline
\end{tabular}

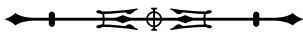


Table 2.

(Continued)

\begin{tabular}{|c|c|c|c|c|c|c|c|c|}
\hline Taxon & Portuguese name & English name & St & PC & $\mathrm{MN}$ & GO & CA & TE \\
\hline Dryocopus lineatus (Linnaeus, 1766) & Pica-pau-de-banda-branca & Lineated Woodpecker & $\mathrm{R}$ & 1,2 & & & u & r \\
\hline Campephilus rubricollis (Boddaert, 1783) & Pica-pau-de-barriga-vermelha & $\begin{array}{l}\text { Red-necked } \\
\text { Woodpecker }\end{array}$ & $\mathrm{R}$ & 1 & & & u & r \\
\hline Campephilus melanoleucos (Gmelin, 1788) & Pica-pau-de-topete-vermelho & $\begin{array}{l}\text { Crimson-crested } \\
\text { Woodpecker }\end{array}$ & $\mathrm{R}$ & & & 1,2 & r & O \\
\hline \multicolumn{9}{|l|}{ Passeriformes Linnaeus, 1758} \\
\hline \multicolumn{9}{|l|}{ Thamnophilidae Swainson, 1824} \\
\hline Cymbilaimus lineatus (Leach, 1814) & Papa-formiga-barrado & Fasciated Antshrike & $\mathrm{R}$ & 1,2 & 1,2 & & c & $r, p$ \\
\hline Taraba major (Vieillot, 1816) & Choró-boi & Great Antshrike & $\mathrm{R}$ & 1,2 & 1,2 & & c & $r, p$ \\
\hline Thamnophilus doliatus (Linnaeus, 1764) & Choca-barrada & Barred Antshrike & $\mathrm{R}$ & & & 1,2 & u & o \\
\hline Thamnophilus schistaceus d'Orbigny, 1835 & Choca-de-olho-vermelho & Plain-winged Antshrike & $\mathrm{R}$ & 1,2 & 1,2 & & c & $r, p$ \\
\hline Thamnophilus aethiops Sclater, 1858 & Choca-lisa & $\begin{array}{l}\text { White-shouldered } \\
\text { Antshrike }\end{array}$ & $\mathrm{R}$ & 1,2 & 1,2 & & c & $r, p$ \\
\hline Neoctantes niger (Pelzeln, 1859) & Choca-preta & Black Bushbird & $\mathrm{R}$ & & 1 & & r & $\mathrm{p}$ \\
\hline $\begin{array}{l}\text { Thamnomanes ardesiacus } \\
\text { (Sclater \& Salvin, 1867) }\end{array}$ & Uirapuru-de-garganta-preta & $\begin{array}{l}\text { Dusky-throated } \\
\text { Antshrike }\end{array}$ & $\mathrm{R}$ & 1,2 & 1,2 & & u & $r, p$ \\
\hline Thamnomanes schistogynus Hellmayr, 1911 & Uirapuru-azul & Bluish-slate Antshrike & $\mathrm{R}$ & 1,2 & 1,2 & & u & $r, p$ \\
\hline Epinecrophylla leucophthalma (Pelzeln, 1868) & Choquinha-de-olho-branco & White-eyed Antwren & $\mathrm{R}$ & 1 & & & r & r \\
\hline Epinecrophylla ornata (Sclater, 1853) & Choquinha-ornada & Ornate Antwren & $\mathrm{R}$ & 1,2 & 1,2 & & r & r \\
\hline Myrmotherula brachyura (Hermann, 1783) & Choquinha-miúda & Pygmy Antwren & $\mathrm{R}$ & 1,2 & & & c & r \\
\hline Myrmotherula sclateri Snethlage, 1912 & $\begin{array}{l}\text { Choquinha-de- } \\
\text { garganta-amarela }\end{array}$ & Sclater's Antwren & $\mathrm{R}$ & 1,2 & & & u & r \\
\hline Myrmotherula hauxwelli (Sclater, 1857) & Choquinha-de-garganta-clara & Plain-throated Antwren & $\mathrm{R}$ & 1,2 & & & u & r \\
\hline Myrmotherula axillaris (Vieillot, 1817) & Choquinha-de-flanco-branco & White-flanked Antwren & $\mathrm{R}$ & 1,2 & & & c & $r, p$ \\
\hline Myrmotherula longipennis Pelzeln, 1868 & Choquinha-de-asa-comprida & Long-winged Antwren & $\mathrm{R}$ & & 1 & & u & $p$ \\
\hline Myrmotherula menetriesii (d'Orbigny, 1837) & Choquinha-de-garganta-cinza & Gray Antwren & $\mathrm{R}$ & 1,2 & & & r & r \\
\hline Microrhopias quixensis (Cornalia, 1849) & Papa-formiga-de-bando & Dot-winged Antwren & $\mathrm{R}$ & 1,2 & 1,2 & & u & r, p \\
\hline $\begin{array}{l}\text { Drymophila devillei } \\
\text { (Menegaux \& Hellmayr, 1906) }\end{array}$ & Trovoada-listrada & Striated Antbird & $\mathrm{R}$ & 1,2 & & & u & $r, p$ \\
\hline Cercomacra cinerascens (Sclater, 1857) & Chororó-pocuá & Gray Antbird & $\mathrm{R}$ & 1,2 & 1,2 & & c & $r, p$ \\
\hline Cercomacra serva (Sclater, 1858) & Chororó-preto & Black Antbird & $\mathrm{R}$ & 1,2 & 1,2 & & c & $r, p$ \\
\hline Myrmoborus leucophrys (Tschudi, 1844) & Papa-formiga-de-sobrancelha & White-browed Antbird & $\mathrm{R}$ & 1,2 & 1,2 & & c & $r, p$ \\
\hline Myrmoborus myotherinus (Spix, 1825) & Formigueiro-de-cara-preta & Black-faced Antbird & $\mathrm{R}$ & 1,2 & 1,2 & & c & $r, p$ \\
\hline Hypocnemis peruviana Taczanowski, 1884 & Cantador-sinaleiro & $\begin{array}{c}\text { Peruvian } \\
\text { Warbling-Antbird }\end{array}$ & $\mathrm{R}$ & & 1,2 & & u & $p$ \\
\hline Hypocnemis subflava Cabanis, 1873 & Cantador-galego & $\begin{array}{l}\text { Yellow-breasted } \\
\text { Warbling-Antbird }\end{array}$ & R\# & 1,2 & 1,2 & & u & $r, p$ \\
\hline
\end{tabular}

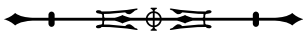


Table 2.

(Continued)

\begin{tabular}{|c|c|c|c|c|c|c|c|c|}
\hline Taxon & Portuguese name & English name & St & PC & $M N$ & GO & CA & TE \\
\hline Percnostola lophotes Hellmayr \& Seilern, 1914 & Formigueiro-do-bambu & White-lined Antbird & $\mathrm{R \#}$ & & 1,2 & & r & $p$ \\
\hline Schistocichla humaythae (Pelzeln, 1868) & Formigueiro-de-cauda-curta & Humaita Antbird & $\mathrm{R}$ & & 1,2 & & r & $\mathrm{p}$ \\
\hline Myrmeciza hemimelaena Sclater, 1857 & $\begin{array}{l}\text { Formigueiro-de-cauda- } \\
\text { castanha }\end{array}$ & Chestnut-tailed Antbird & $\mathrm{R}$ & 1,2 & 1,2 & & c & $r, p$ \\
\hline Myrmeciza goeldii (Snethlage, 1908) & Formigueiro-de-goeldi & Goeldi's Antbird & $\mathrm{R}$ & 1,2 & 1,2 & & c & $r, p$ \\
\hline Myrmeciza hyperythra (Sclater, 1855) & Formigueiro-chumbo & Plumbeous Antbird & $\mathrm{R}$ & & 1,2 & & r & $\mathrm{p}$ \\
\hline Myrmeciza fortis (Sclater \& Salvin, 1868) & Formigueiro-de-taoca & Sooty Antbird & $\mathrm{R}$ & & 1,2 & & u & $\mathrm{p}$ \\
\hline Gymnopithys salvini (Berlepsch, 1901) & $\begin{array}{l}\text { Mãe-de-taoca-de-cauda- } \\
\text { barrada }\end{array}$ & White-throated Antbird & $\mathrm{R}$ & & 1,2 & & u & $\mathrm{p}$ \\
\hline $\begin{array}{l}\text { Rhegmatorhina melanosticta } \\
\text { (Sclater \& Salvin, 1880) }\end{array}$ & Mãe-de-taoca-cabeçuda & Hairy-crested Antbird & $\mathrm{R}$ & & 1,2 & & r & $\mathrm{p}$ \\
\hline Hylophylax naevius (Gmelin, 1789) & Guarda-floresta & Spot-backed Antbird & $\mathrm{R}$ & 1,2 & 1,2 & & u & $r, p$ \\
\hline Willisornis poecilinotus (Cabanis, 1847) & Rendadinho & Scale-backed Antbird & $\mathrm{R}$ & 1,2 & 1,2 & & u & $r, p$ \\
\hline $\begin{array}{l}\text { Phlegopsis nigromaculata } \\
\text { (d'Orbigny \& Lafresnaye, 1837) }\end{array}$ & Mãe-de-taoca & Black-spotted Bare-eye & $\mathrm{R}$ & 1,2 & 1,2 & & c & $r, p$ \\
\hline \multicolumn{9}{|l|}{ Conopophagidae Sclater \& Salvin, 1873} \\
\hline Conopophaga peruviana Des Murs, 1856 & Chupa-dente-do-peru & Ash-throated Gnateater & $\mathrm{R}$ & & 1 & & r & $\mathrm{p}$ \\
\hline \multicolumn{9}{|l|}{ Grallariidae Sclater \& Salvin, 1873} \\
\hline Myrmothera campanisona (Hermann, 1783) & Tovaca-patinho & Thrush-like Antpitta & $\mathrm{R}$ & 1,2 & 1,2 & & u & r \\
\hline \multicolumn{9}{|l|}{ Rhinocryptidae Wetmore, 1930} \\
\hline Liosceles thoracicus (Sclater, 1865) & Corneteiro-da-mata & Rusty-belted Tapaculo & $\mathrm{R}$ & 2 & & & r & r \\
\hline \multicolumn{9}{|l|}{ Formicariidae Gray, 1840} \\
\hline Formicarius colma Boddaert, 1783 & Galinha-do-mato & $\begin{array}{l}\text { Rufous-capped } \\
\text { Antthrush }\end{array}$ & $\mathrm{R}$ & 1,2 & 1,2 & & $c$ & $r, p$ \\
\hline $\begin{array}{l}\text { Formicarius analis } \\
\text { (d'Orbigny \& Lafresnaye, 1837) }\end{array}$ & $\begin{array}{l}\text { Pinto-do-mato-de- } \\
\text { cara-preta }\end{array}$ & Black-faced Antthrush & $\mathrm{R}$ & 1,2 & 1,2 & & c & $r, p$ \\
\hline \multicolumn{9}{|l|}{ Scleruridae Swainson, 1827} \\
\hline Sclerurus mexicanus Sclater, 1857 & Vira-folha-de-peito-vermelho & $\begin{array}{l}\text { Tawny-throated } \\
\text { Leaftosser }\end{array}$ & $\mathrm{R}$ & & 1 & & r & $\mathrm{p}$ \\
\hline Sclerurus caudacutus (Vieillot, 1816) & Vira-folha-pardo & Black-tailed Leaftosser & $\mathrm{R}$ & & 1 & & r & $\mathrm{p}$ \\
\hline \multicolumn{9}{|l|}{ Dendrocolaptidae Gray, 1840} \\
\hline Dendrocincla fuliginosa (Vieillot, 1818) & Arapaçu-pardo & $\begin{array}{l}\text { Plain-brown } \\
\text { Woodcreeper }\end{array}$ & $\mathrm{R}$ & 1,2 & 1,2 & & c & $r, p$ \\
\hline Dendrocincla merula (Lichtenstein, 1829) & Arapaçu-da-taoca & $\begin{array}{l}\text { White-chinned } \\
\text { Woodcreeper }\end{array}$ & $\mathrm{R}$ & & 1,2 & & c & $\mathrm{p}$ \\
\hline Deconychura longicauda (Pelzeln, 1868) & Arapaçu-rabudo & $\begin{array}{l}\text { Long-tailed } \\
\text { Woodcreeper }\end{array}$ & $\mathrm{R}$ & & 1,2 & & u & $\mathrm{p}$ \\
\hline
\end{tabular}

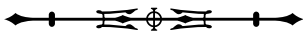


Table 2.

(Continued)

\begin{tabular}{|c|c|c|c|c|c|c|c|c|}
\hline Taxon & Portuguese name & English name & St & PC & $\mathrm{MN}$ & GO & CA & TE \\
\hline Sittasomus griseicapillus (Vieillot, 1818) & Arapaçu-verde & $\begin{array}{c}\text { Olivaceous } \\
\text { Woodcreeper }\end{array}$ & $\mathrm{R}$ & 1,2 & 1,2 & & C & $r, p$ \\
\hline Glyphorynchus spirurus (Vieillot, 1819) & Arapaçu-de-bico-de-cunha & $\begin{array}{l}\text { Wedge-billed } \\
\text { Woodcreeper }\end{array}$ & $\mathrm{R}$ & & 1,2 & & c & P \\
\hline Dendrexetastes rufigula (Lesson, 1844) & Arapaçu-galinha & $\begin{array}{l}\text { Cinnamon-throated } \\
\text { Woodcreeper }\end{array}$ & $\mathrm{R}$ & 2 & & & r & r \\
\hline Hylexetastes stresemanni Snethlage, 1925 & Arapaçu-de-barriga-pintada & $\begin{array}{l}\text { Bar-bellied } \\
\text { Woodcreeper }\end{array}$ & $\mathrm{R}$ & & 2 & & r & $r, p$ \\
\hline $\begin{array}{l}\text { Xiphocolaptes promeropirhynchus } \\
\text { (Lesson, 1840) }\end{array}$ & Arapaçu-vermelho & $\begin{array}{l}\text { Strong-billed } \\
\text { Woodcreeper }\end{array}$ & $\mathrm{R}$ & & & 1,2 & r & r \\
\hline Dendrocolaptes certhia (Boddaert, 1783) & Arapaçu-barrado & $\begin{array}{l}\text { Amazonian Barred- } \\
\text { Woodcreeper }\end{array}$ & $\mathrm{R}$ & 1,2 & 1,2 & & u & $r, p$ \\
\hline Dendrocolaptes picumnus Lichtenstein, 1820 & Arapaçu-meio-barrado & $\begin{array}{l}\text { Black-banded } \\
\text { Woodcreeper }\end{array}$ & $\mathrm{R}$ & 1,2 & 1,2 & & u & $r, p$ \\
\hline Xiphorhynchus chunchotambo (Tschudi, 1844) & Arapaçu-de-tschudi & Tschudi's Woodcreeper & $\mathrm{R \#}$ & 1,2 & 1,2 & & u & r, p \\
\hline Xiphorhynchus elegans (Pelzeln, 1868) & Arapaçu-elegante & Elegant Woodcreeper & $\mathrm{R}$ & 1,2 & 1,2 & & u & $r, p$ \\
\hline Xiphorhynchus guttatus (Lichtenstein, 1820) & Arapaçu-de-garganta-amarela & $\begin{array}{l}\text { Buff-throated } \\
\text { Woodcreeper }\end{array}$ & $\mathrm{R}$ & 1,2 & 1,2 & & c & $r, p$ \\
\hline $\begin{array}{l}\text { Campylorhamphus trochilirostris } \\
\text { (Lichtenstein, 1820) }\end{array}$ & Arapaçu-beija-flor & Red-billed Scythebill & $\mathrm{R}$ & & 1,2 & & u & $p, c$ \\
\hline \multicolumn{9}{|l|}{ Furnariidae Gray, 1840} \\
\hline Furnarius leucopus Swainson, 1838 & Casaca-de-couro-amarelo & Pale-legged Hornero & $\mathrm{R}$ & & & 3 & c & ○ \\
\hline Synallaxis rutilans Temminck, 1823 & João-teneném-castanho & Ruddy Spinetail & $\mathrm{R}$ & 1,2 & 2 & & c & r, p \\
\hline Synallaxis gujanensis (Gmelin, 1789) & João-teneném-becuá & Plain-crowned Spinetail & $\mathrm{R}$ & & 2 & & u & p \\
\hline Simoxenops ucayalae (Chapman, 1928) & $\begin{array}{l}\text { Limpa-folha-de- } \\
\text { bico-virado }\end{array}$ & Peruvian Recurvebill & $\mathrm{R}$ & & 1,2 & & r & $P$ \\
\hline Hyloctistes subulatus (Spix, 1824) & Limpa-folha-riscado & Striped Woodhaunter & $\mathrm{R}$ & & 1 & & r & $\mathrm{p}$ \\
\hline $\begin{array}{l}\text { Philydor ruficaudatum } \\
\text { (d'Orbigny \& Lafresnaye, 1838) }\end{array}$ & Limpa-folha-de-cauda-ruiva & $\begin{array}{l}\text { Rufous-Tailed } \\
\text { Foliage-gleaner }\end{array}$ & $\mathrm{R}$ & 1 & 1 & & u & $P$ \\
\hline Philydor erythrocercum (Pelzeln, 1859) & Limpa-folha-de-sobre-ruivo & $\begin{array}{l}\text { Rufous-rumped } \\
\text { Foliage-gleaner }\end{array}$ & $\mathrm{R}$ & 1 & 1 & & u & $P$ \\
\hline Philydor erythropterum (Sclater, 1856) & Limpa-folha-de-asa-castanha & $\begin{array}{l}\text { Chestnut-winged } \\
\text { Foliage-gleaner }\end{array}$ & $\mathrm{R}$ & & 1,2 & & u & $P$ \\
\hline Philydor pyrrhodes (Cabanis, 1848) & Limpa-folha-vermelho & $\begin{array}{l}\text { Cinnamon-rumped } \\
\text { Foliage-gleaner }\end{array}$ & $\mathrm{R}$ & & & 1,2 & u & O \\
\hline Automolus ochrolaemus (Tschudi, 1844) & Barranqueiro-camurça & $\begin{array}{l}\text { Buff-throated } \\
\text { Foliage-gleaner }\end{array}$ & $\mathrm{R}$ & 1,2 & 1,2 & & c & $r, p$ \\
\hline
\end{tabular}

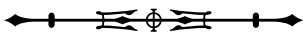


Table 2.

(Continued)

\begin{tabular}{|c|c|c|c|c|c|c|c|c|}
\hline Taxon & Portuguese name & English name & St & PC & $M N$ & GO & CA & TE \\
\hline Automolus infuscatus (Sclater, 1856) & Barranqueiro-pardo & $\begin{array}{l}\text { Olive-backed } \\
\text { Foliage-gleaner }\end{array}$ & $\mathrm{R}$ & 1,2 & 1,2 & & $c$ & $r, p$ \\
\hline Automolus melanopezus (Sclater, 1858) & Barranqueiro-escuro & $\begin{array}{l}\text { Brown-rumped } \\
\text { Foliage-gleaner }\end{array}$ & $\mathrm{R}$ & & 1 & & u & $\mathrm{p}$ \\
\hline Automolus rubiginosus (Sclater, 1857) & Barranqueiro-ferrugem & Ruddy Foliage-gleaner & $\mathrm{R}$ & 1,2 & 1,2 & & r & r \\
\hline Xenops tenuirostris Pelzeln, 1859 & Bico-virado-fino & Slender-billed Xenops & $\mathrm{R}$ & 1 & & & r & r \\
\hline Xenops minutus (Sparrman, 1788) & Bico-virado-miúdo & Plain Xenops & $\mathrm{R}$ & & 1,2 & & $c$ & p \\
\hline \multicolumn{9}{|l|}{ Tyrannidae Vigors, 1825} \\
\hline Mionectes oleagineus (Chubb, 1919) & Abre-asa-da-mata & $\begin{array}{l}\text { MacConnell's } \\
\text { Flycatcher }\end{array}$ & $\mathrm{R}$ & & 1,2 & & u & $\mathrm{P}$ \\
\hline Leptopogon amaurocephalus Tschudi, 1846 & Cabeçudo & $\begin{array}{l}\text { Sepia-capped } \\
\text { Flycatcher }\end{array}$ & $\mathrm{R}$ & & 1,2 & & u & $p$ \\
\hline Corythopis torquatus (Tschudi, 1844) & Estalador-do-norte & Ringed Antpipit & $\mathrm{R}$ & & 1,2 & & u & $\mathrm{p}$ \\
\hline Lophotriccus eulophotes Todd, 1925 & Maria-topetuda & $\begin{array}{l}\text { Long-crested } \\
\text { Pygmy-Tyrant }\end{array}$ & $\mathrm{R}$ & 1,2 & 1,2 & & r & $\mathrm{p}$ \\
\hline Hemitriccus minor (Snethlage, 1907) & Maria-sebinha & Snethlage's Tody-Tyrant & $\mathrm{R}$ & & 1,2 & & u & $\mathrm{p}$ \\
\hline Hemitriccus flammulatus Berlepsch, 1901 & Maria-de-peito-machetado & $\begin{array}{l}\text { Flammulated } \\
\text { Bamboo-Tyrant }\end{array}$ & $\mathrm{R}$ & 1,2 & 1,2 & & u & $\mathrm{p}$ \\
\hline Hemitriccus griseipectus (Snethlage, 1907) & Maria-de-barriga-branca & $\begin{array}{l}\text { White-bellied } \\
\text { Tody-Tyrant }\end{array}$ & $\mathrm{R}$ & 1 & & & r & r \\
\hline Hemitriccus minimus (Todd, 1925) & Maria-mirim & Zimmer's Tody-Tyrant & $\mathrm{R}$ & 1,2 & & & u & r \\
\hline $\begin{array}{l}\text { Myiornis ecaudatus } \\
\text { (d'Orbigny \& Lafresnaye, 1837) }\end{array}$ & Caçula & $\begin{array}{l}\text { Short-tailed } \\
\text { Pygmy-Tyrant }\end{array}$ & $\mathrm{R}$ & 1,2 & & & $c$ & r \\
\hline Todirostrum maculatum (Desmarest, 1806) & Ferreirinho-estriado & $\begin{array}{c}\text { Spotted } \\
\text { Tody-Flycatcher }\end{array}$ & $\mathrm{R}$ & & & 1,2 & u & O \\
\hline Todirostrum chrysocrotaphum Strickland, 1850 & Ferreirinho-pintado & $\begin{array}{l}\text { Yellow-browed } \\
\text { Tody-Flycatcher }\end{array}$ & $\mathrm{R}$ & & & 1,2 & u & O \\
\hline Tyrannulus elatus (Latham, 1790) & Maria-te-viu & $\begin{array}{l}\text { Yellow-crowned } \\
\text { Tyrannulet }\end{array}$ & $\mathrm{R}$ & 1,2 & & & r & r \\
\hline Myiopagis gaimardii (d'Orbigny, 1839) & Maria-pechim & Forest Elaenia & $\mathrm{R}$ & 1,2 & & & c & r \\
\hline Myiopagis caniceps (Swainson, 1835) & Guaracava-cinzenta & Gray Elaenia & $\mathrm{R}$ & 1,2 & & & u & r \\
\hline Elaenia flavogaster (Thunberg, 1822) & $\begin{array}{l}\text { Guaracava-de- } \\
\text { barriga-amarela }\end{array}$ & Yellow-bellied Elaenia & $\mathrm{R}$ & & & 2,3 & u & O \\
\hline Elaenia parvirostris Pelzeln, 1868 & Guaracava-de-bico-curto & Small-billed Elaenia & $\mathrm{R}$ & & 1,2 & & u & $\mathrm{p}$ \\
\hline Ornithion inerme Hartlaub, 1853 & Poiaeiro-de-sobrancelha & White-lored Tyrannulet & $\mathrm{R}$ & 1,2 & & & u & r \\
\hline Camptostoma obsoletum (Temminck, 1824) & Risadinha & $\begin{array}{c}\text { Southern } \\
\text { Beardless-Tyrannulet }\end{array}$ & $\mathrm{R}$ & & & 1,2 & u & O \\
\hline
\end{tabular}

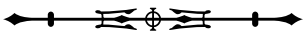


Table 2.

(Continued)

\begin{tabular}{|c|c|c|c|c|c|c|c|c|}
\hline Taxon & Portuguese name & English name & St & PC & $M N$ & GO & CA & TE \\
\hline Phaeomyias murina (Spix, 1825) & Bagageiro & $\begin{array}{c}\text { Mouse-colored } \\
\text { Tyrannulet }\end{array}$ & $\mathrm{R}$ & & & 1,2 & u & O \\
\hline Zimmerius gracilipes (Sclater \& Salvin, 1868) & Poiaeiro-de-pata-fina & $\begin{array}{l}\text { Slender-footed } \\
\text { Tyrannulet }\end{array}$ & $\mathrm{R}$ & 1,2 & & & u & r \\
\hline Cnipodectes superrufus Lane et al., 2007 & Flautim-rufo & Rufous Twistwing & R\# & & 1,2 & & r & $p, c$ \\
\hline $\begin{array}{l}\text { Tolmomyias poliocephalus } \\
\text { (Taczanowski, 1884) }\end{array}$ & Bico-chato-de-cabeça-cinza & $\begin{array}{l}\text { Gray-crowned } \\
\text { Flycatcher }\end{array}$ & $\mathrm{R}$ & 1,2 & 1,2 & & c & $r, p$ \\
\hline Tolmomyias flaviventris (Wied, 1831) & Bico-chato-amarelo & $\begin{array}{l}\text { Yellow-breasted } \\
\text { Flycatcher }\end{array}$ & $\mathrm{R}$ & 1,2 & 1,2 & & c & $r, p$ \\
\hline Platyrinchus coronatus Sclater, 1858 & Patinho-de-coroa-dourada & $\begin{array}{l}\text { Golden-crowned } \\
\text { Spadebill }\end{array}$ & $\mathrm{R}$ & & 1,2 & & u & $r, p$ \\
\hline Platyrinchus platyrhynchos (Gmelin, 1788) & Patinho-de-coroa-branca & White-crested Spadebill & $\mathrm{R}$ & 1,2 & 1,2 & & c & $r, p$ \\
\hline $\begin{array}{l}\text { Onychorhynchus coronatus } \\
\text { (Statius Muller, 1776) }\end{array}$ & Maria-leque & Royal Flycatcher & $\mathrm{R}$ & 1,2 & 1,2 & & c & $r, p$ \\
\hline Terenotriccus erythrurus (Cabanis, 1847) & Papa-moscas-uirapuru & Ruddy-tailed Flycatcher & $\mathrm{R}$ & 1,2 & 1,2 & & c & $r, p$ \\
\hline Lathrotriccus euleri (Cabanis, 1868) & Enferrujado & Euler's Flycatcher & $\mathrm{R}$ & 1,2 & 1,2 & & c & $r, p$ \\
\hline Empidonax cf. alnorum Brewster, 1895 & Papa-moscas-de-alder & Alder Flycatcher & VN\# & & 1,2 & & r & $\mathrm{p}$ \\
\hline Contopus cooperi (Nuttall, 1831) & Piui-boreal & Olive-sided Flycatcher & VN & & & 1,2 & r & O \\
\hline Contopus virens (Linnaeus, 1766) & Piui-verdadeiro & Eastern Wood-Pewee & VN\# & & & 1,2 & r & ० \\
\hline Pyrocephalus rubinus (Boddaert, 1783) & Príncipe & Vermilion Flycatcher & $\mathrm{R}$ & & & 1,2 & r & O \\
\hline Colonia colonus (Vieillot, 1818) & Viuvinha & Long-tailed Tyrant & $\mathrm{R}$ & & & 1,2 & r & O \\
\hline Legatus leucophaius (Vieillot, 1818) & Bem-te-vi-pirata & Piratic Flycatcher & $\mathrm{R}$ & 1,2 & & & c & $r$ \\
\hline Myiozetetes cayanensis (Linnaeus, 1766) & $\begin{array}{l}\text { Bentevizinho-de-asa- } \\
\text { ferrugínea }\end{array}$ & $\begin{array}{l}\text { Rusty-margined } \\
\text { Flycatcher }\end{array}$ & $\mathrm{R}$ & & & 1,2 & c & ○ \\
\hline Myiozetetes luteiventris (Sclater, 1858) & Bem-te-vi-barulhento & Dusky-chested Flycatcher & $\mathrm{R}$ & & & 1,2 & r & O \\
\hline Pitangus sulphuratus (Linnaeus, 1766) & Bem-te-vi & Great Kiskadee & $\mathrm{R}$ & & & 1,2 & c & r \\
\hline Philohydor lictor (Lichtenstein, 1823) & Bentevizinho-do-brejo & Lesser Kiskadee & $\mathrm{R}$ & & & 1,2 & u & o \\
\hline Myiodynastes maculatus (Statius Muller, 1776) & Bem-te-vi-rajado & Streaked Flycatcher & $\mathrm{R}$ & 1,2 & & & c & $\mathrm{p}$ \\
\hline Megarynchus pitangua (Linnaeus, 1766) & Neinei & Boat-billed Flycatcher & $\mathrm{R}$ & 1,2 & & & c & r \\
\hline Empidonomus varius (Vieillot, 1818) & Peitica & Variegated Flycatcher & $\mathrm{R}$ & & & 1,2 & c & o \\
\hline Tyrannus melancholicus Vieillot, 1819 & Suiriri & Tropical Kingbird & $\mathrm{R}$ & & & 2,3 & c & $r$ \\
\hline Tyrannus savana Vieillot, 1808 & Tesourinha & Fork-tailed Flycatcher & $\mathrm{R}$ & & & 2,3 & u & o \\
\hline Tyrannus tyrannus (Linnaeus, 1766) & Suiriri-valente & Eastern Kingbird & VN & & & 2,3 & u & O \\
\hline Rhytipterna simplex (Lichtenstein, 1823) & Vissiá & Grayish Mourner & $\mathrm{R}$ & 1,2 & 1,2 & & c & r \\
\hline Sirystes sibilator (Vieillot, 1818) & Gritador & Sirystes & $R$ & 1,2 & & & c & $r$ \\
\hline $\begin{array}{l}\text { Myiarchus tuberculifer } \\
\text { (d'Orbigny \& Lafresnaye, 1837) }\end{array}$ & Maria-cavaleira-pequena & $\begin{array}{l}\text { Dusky-capped } \\
\text { Flycatcher }\end{array}$ & $\mathrm{R}$ & & & 1,2 & u & O \\
\hline
\end{tabular}


Table 2.

(Continued)

\begin{tabular}{|c|c|c|c|c|c|c|c|c|}
\hline Taxon & Portuguese name & English name & St & PC & $M N$ & GO & CA & $\mathrm{TE}$ \\
\hline Myiarchus swainsoni Cabanis \& Heine, 1859 & Irré & Swainson's Flycatcher & $\mathrm{R}$ & & & 1,2 & $\mathrm{u}$ & o \\
\hline Myiarchus ferox (Gmelin, 1789) & Maria-cavaleira & Short-crested Flycatcher & $\mathrm{R}$ & & & 2,3 & u & o \\
\hline Ramphotrigon megacephalum (Swainson, 1835) & Maria-cabeçuda & Large-headed Flatbill & $\mathrm{R}$ & 2,3 & 2,3 & & c & $r, p$ \\
\hline Ramphotrigon ruficauda (Spix, 1825) & Bico-chato-de-rabo-vermelho & Rufous-tailed Flatbill & $\mathrm{R}$ & 2,3 & & & u & r \\
\hline Ramphotrigon fuscicauda Chapman, 1925 & Maria-de-cauda-escura & Dusky-tailed Flatbill & $\mathrm{R}$ & & 2,3 & & u & $p, c$ \\
\hline Attila spadiceus (Gmelin, 1789) & Capitão-de-saíra-amarelo & Bright-rumped Attila & $\mathrm{R}$ & 2,3 & 2,3 & & c & $\mathrm{r}, \mathrm{c}$ \\
\hline \multicolumn{9}{|l|}{ Cotingidae Bonaparte, 1849} \\
\hline Cotinga cayana (Linnaeus, 1766) & Anambé-azul & Spangled Cotinga & $\mathrm{R}$ & & 1 & & u & $\mathrm{p}$ \\
\hline Lipaugus vociferans (Wied, 1820) & Cricrió & Screaming Piha & $\mathrm{R}$ & 1,2 & 1 & & c & $r, p$ \\
\hline Conioptilon mcilhennyi Lowery \& O’Neill, 1966 & Anambé-de-cara-preta & Black-faced Cotinga & R\# & 1,2 & & & u & r \\
\hline Gymnoderus foetidus (Linnaeus, 1758) & Anambé-pombo & Bare-necked Fruitcrow & $\mathrm{R}$ & 1,2 & & & r & o \\
\hline Querula purpurata (Statius Muller, 1776) & Anambé-una & $\begin{array}{l}\text { Purple-throated } \\
\text { Fruitcrow }\end{array}$ & $\mathrm{R}$ & 1,2 & & & $c$ & r \\
\hline \multicolumn{9}{|l|}{ Pipridae Rafinesque, 1815} \\
\hline Neopelma sulphureiventer (Hellmayr, 1903) & Fruxu-de-barriga-amarela & $\begin{array}{l}\text { Sulphur-bellied } \\
\text { Tyrant-Manakin }\end{array}$ & $\mathrm{R}$ & 1,2 & & & r & $\mathrm{p}$ \\
\hline Tyranneutes stolzmanni (Hellmayr, 1906) & Uirapuruzinho & Dwarf Tyrant-Manakin & $\mathrm{R}$ & & 1,2 & & c & r \\
\hline Piprites chloris (Temminck, 1822) & Papinho-amarelo & Wing-barred Piprites & $\mathrm{R}$ & & 1,2 & & u & r \\
\hline Machaeropterus pyrocephalus (Sclater, 1852) & Uirapuru-cigarra & Fiery-capped Manakin & $\mathrm{R}$ & 1,2 & & & c & r \\
\hline Lepidothrix coronata (Spix, 1825) & Uirapuru-de-chapéu-azul & Blue-crowned Mankin & $\mathrm{R}$ & 1,2 & 1,2 & & c & $r, p$ \\
\hline Chiroxiphia pareola (Linnaeus, 1766) & Tangará-falso & Blue-backed Manakin & $\mathrm{R}$ & 1 & 1 & & c & $r, p$ \\
\hline Pipra fasciicauda Hellmayr, 1906 & Uirapuru-laranja & Band-tailed Manakin & $\mathrm{R}$ & 1,2 & & & c & $r, p$ \\
\hline Pipra rubrocapilla Temminck, 1821 & Cabeça-encarnada & Red-headed Manakin & $\mathrm{R}$ & 1,2 & 1,2 & & c & $r, p$ \\
\hline \multicolumn{9}{|l|}{ Tityridae Gray, 1840} \\
\hline Iodopleura isabellae Parzudaki, 1847 & Anambé-de-coroa & $\begin{array}{l}\text { White-browed } \\
\text { Purpletuft }\end{array}$ & $\mathrm{R}$ & 2,3 & & & u & r \\
\hline Tityra inquisitor (Lichtenstein, 1823) & $\begin{array}{l}\text { Anambé-branco-de- } \\
\text { bochecha-parda }\end{array}$ & Black-crowned Tityra & $\mathrm{R}$ & & & 1,2 & u & o \\
\hline Tityra cayana (Linnaeus, 1766) & $\begin{array}{l}\text { Anambé-branco-de- } \\
\text { rabo-preto }\end{array}$ & Black-tailed Tityra & $\mathrm{R}$ & 1,2 & & & c & r \\
\hline Tityra semifasciata (Spix, 1825) & $\begin{array}{l}\text { Anambé-branco-de- } \\
\text { máscara-negra }\end{array}$ & Masked Tityra & $\mathrm{R}$ & & & 1,2 & u & o \\
\hline $\begin{array}{l}\text { Pachyramphus castaneus } \\
\text { (Jardine \& Selby, 1827) }\end{array}$ & Caneleiro & $\begin{array}{c}\text { Chestnut-crowned } \\
\text { Becard }\end{array}$ & $\mathrm{R}$ & 1,2 & & & r & r \\
\hline Pachyramphus polychopterus (Vieillot, 1818) & Caneleiro-preto & White-winged Becard & $\mathrm{R}$ & 1,2 & 1,2 & & u & $r, p$ \\
\hline Pachyramphus marginatus (Lichtenstein, 1823) & Caneleiro-bordado & Black-capped Becard & $\mathrm{R}$ & & & 1,2 & $\mathrm{u}$ & o \\
\hline Pachyramphus minor (Lesson, 1830) & Caneleiro-pequeno & Pink-throated Becard & $\mathrm{R}$ & 1,2 & & & $\mathrm{u}$ & r \\
\hline
\end{tabular}


Table 2.

(Continued)

\begin{tabular}{|c|c|c|c|c|c|c|c|c|}
\hline Taxon & Portuguese name & English name & St & PC & $\mathrm{MN}$ & GO & CA & TE \\
\hline \multicolumn{9}{|l|}{ Vireonidae Swainson, 1837} \\
\hline Cyclarhis gujanensis (Gmelin, 1789) & Pitiguari & $\begin{array}{l}\text { Rufous-browed } \\
\text { Peppershrike }\end{array}$ & $\mathrm{R}$ & 1,2 & & & c & r \\
\hline Vireolanius leucotis (Swainson, 1838) & Assobiador-do-castanhal & $\begin{array}{l}\text { Slaty-capped } \\
\text { Shrike-Vireo }\end{array}$ & $\mathrm{R}$ & 1,2 & & & c & r \\
\hline Vireo olivaceus (Linnaeus, 1766) & Juruviara & Red-eyed Vireo & $\mathrm{R}$ & & & 1,2 & u & o \\
\hline Hylophilus thoracicus Temminck, 1822 & Vite-vite & $\begin{array}{l}\text { Lemon-chested } \\
\text { Greenlet }\end{array}$ & $\mathrm{R}$ & 1,2 & & & u & 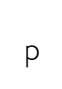 \\
\hline Hylophilus hypoxanthus Pelzeln, 1868 & Vite-vite-de-barriga-marela & $\begin{array}{l}\text { Dusky-capped } \\
\text { Greenlet }\end{array}$ & $\mathrm{R}$ & 1,2 & & & u & $p$ \\
\hline Hylophilus ochraceiceps Sclater, 1860 & Vite-vite-uirapuru & $\begin{array}{c}\text { Tawny-crowned } \\
\text { Greenlet }\end{array}$ & $\mathrm{R}$ & & 1 & & u & O \\
\hline \multicolumn{9}{|l|}{ Hirundinidae Rafinesque, 1815} \\
\hline Pygochelidon cyanoleuca (Vieillot, 1817) & Andorinha-pequena-de-casa & $\begin{array}{l}\text { Blue-and-white } \\
\text { Swallow }\end{array}$ & $\mathrm{R}$ & & & 4 & u & O \\
\hline Stelgidopteryx ruficollis (Vieillot, 1817) & Andorinha-serradora & $\begin{array}{l}\text { Southern Rough- } \\
\text { winged Swallow }\end{array}$ & $\mathrm{R}$ & & & 4 & u & O \\
\hline Progne tapera (Vieillot, 1817) & Andorinha-do-campo & Brown-chested Martin & $\mathrm{R}$ & & & 4 & u & o \\
\hline Progne subis (Linnaeus, 1758) & Andorinha-azul & Purple Martin & VN & & & 4 & u & o \\
\hline Tachycineta albiventer (Boddaert, 1783) & Andorinha-do-rio & White-winged Swallow & $\mathrm{R}$ & & & 4 & u & o \\
\hline \multicolumn{9}{|l|}{ Troglodytidae Swainson, 1831} \\
\hline Microcerculus marginatus (Sclater, 1855) & Uirapuru-veado & Scaly-breasted Wren & $\mathrm{R}$ & & 1,2 & & r & $\mathrm{p}$ \\
\hline Troglodytes musculus Naumann, 1823 & Corruíra & Southern House-Wren & $\mathrm{R}$ & & & 1,2 & c & r \\
\hline Campylorhynchus turdinus (Wied, 1831) & Catatau & Thrush-like Wren & $\mathrm{R}$ & & & 1,2 & u & o \\
\hline Pheugopedius genibarbis (Swainson, 1838) & Garrinchão-pai-avô & Moustached Wren & $\mathrm{R}$ & 1,2 & 2 & & c & $r, p$ \\
\hline \multicolumn{9}{|l|}{ Polioptilidae Baird, 1858} \\
\hline Ramphocaenus melanurus Vieillot, 1819 & Bico-assovelado & Long-billed Gnatwren & $\mathrm{R}$ & & 1,2 & & u & $p$ \\
\hline \multicolumn{9}{|l|}{ Turdidae Rafinesque, 1815} \\
\hline Catharus ustulatus (Nuttall, 1840) & Sabiá-de-óculos & Swainson's Thrush & VN & & 1,2 & & u & $\mathrm{p}$ \\
\hline Turdus hauxwelli Lawrence, 1869 & Sabiá-bicolor & Hauxwell's Thrush & $\mathrm{R}$ & & 1,2 & & u & $p, c$ \\
\hline Turdus albicollis Vieillot, 1818 & Sabiá-coleira & White-necked Thrush & $\mathrm{R}$ & & 1,2 & & c & $\mathrm{p}$ \\
\hline \multicolumn{9}{|l|}{ Thraupidae Cabanis, 1847} \\
\hline Saltator grossus (Linnaeus, 1766) & Bico-encarnado & Slate-colored Grosbeak & $\mathrm{R}$ & 1,2 & 1,2 & & c & $r, p$ \\
\hline Saltator maximus (Statius Muller, 1776) & Tempera-viola & Buff-throated Saltator & $\mathrm{R}$ & 1,2 & 1,2 & & u & r \\
\hline Saltator coerulescens Vieillot, 1817 & Sabiá-gongá & Grayish Saltator & $\mathrm{R}$ & 1,2 & & & r & r \\
\hline Cissopis leverianus (Gmelin, 1788) & Tietinga & Magpie Tanager & $\mathrm{R}$ & & & 2,3 & u & o \\
\hline Lamprospiza melanoleuca (Vieillot, 1817) & Pipira-de-bico-vermelho & Red-billed Pied Tanager & $\mathrm{R}$ & 1,2 & & & c & r \\
\hline
\end{tabular}


Table 2.

(Continued)

\begin{tabular}{|c|c|c|c|c|c|c|c|c|}
\hline Taxon & Portuguese name & English name & St & PC & $M N$ & $\mathrm{GO}$ & CA & TE \\
\hline Eucometis penicillata (Spix, 1825) & Pipira-da-taoca & Gray-headed Tanager & $\mathrm{R}$ & & & 2 & u & o \\
\hline Tachyphonus cristatus (Linnaeus, 1766) & Tiê-galo & Flame-crested Tanager & $\mathrm{R}$ & 1,2 & & & u & r \\
\hline $\begin{array}{l}\text { Tachyphonus luctuosus d'Orbigny \& } \\
\text { Lafresnaye, } 1837\end{array}$ & Tem-tem-de-dragona-branca & $\begin{array}{l}\text { White-shouldered } \\
\text { Tanager }\end{array}$ & $\mathrm{R}$ & 1,2 & 1,2 & & $\mathrm{u}$ & $r, p$ \\
\hline $\begin{array}{l}\text { Lanio versicolor } \\
\text { (d'Orbigny \& Lafresnaye, 1837) }\end{array}$ & Pipira-de-asa-branca & $\begin{array}{l}\text { White-winged } \\
\text { Shrike-Tanager }\end{array}$ & $\mathrm{R}$ & & & 1,2 & u & O \\
\hline Ramphocelus carbo (Pallas, 1764) & Pipira-vermelha & Silver-beaked Tanager & $\mathrm{R}$ & & & 2,3 & c & ० \\
\hline Thraupis episcopus (Linnaeus, 1766) & Sanhaçu-da-amazônia & Blue-gray Tanager & $\mathrm{R}$ & & & 2,3 & c & O \\
\hline Thraupis palmarum (Wied, 1823) & Sanhaçu-do-coqueiro & Palm Tanager & $\mathrm{R}$ & & & 2,3 & u & o \\
\hline Tangara chilensis (Vigors, 1832) & Sete-cores-da-amazônia & Paradise Tanager & $\mathrm{R}$ & 1,2 & & & u & r \\
\hline Tangara schrankii (Spix, 1825) & Saíra-ouro & $\begin{array}{l}\text { Green-and-gold } \\
\text { Tanager }\end{array}$ & $\mathrm{R}$ & 1,2 & & & $\mathrm{u}$ & r \\
\hline Tangara nigrocincta (Bonaparte, 1838) & Saíra-mascarada & Masked Tanager & $\mathrm{R}$ & 1,2 & & & $\mathrm{u}$ & $r$ \\
\hline Tangara velia (Linnaeus, 1758) & Saíra-diamante & Opal-rumped Tanager & $\mathrm{R}$ & 1,2 & & & $\mathrm{u}$ & r \\
\hline Tangara callophrys (Cabanis, 1849) & Saíra-opala & Opal-crowned Tanager & $\mathrm{R}$ & 1,2 & & & $\mathrm{u}$ & r \\
\hline Dacnis lineata (Gmelin, 1789) & Saí-de-máscara-preta & Black-faced Dacnis & $\mathrm{R}$ & 1,2 & & & $\mathrm{u}$ & r \\
\hline Dacnis cayana (Linnaeus, 1766) & Saí-azul & Blue Dacnis & $\mathrm{R}$ & 1,2 & & & u & r \\
\hline Chlorophanes spiza (Linnaeus, 1758) & Saí-verde & Green Honeycreeper & $\mathrm{R}$ & & & 1,2 & u & O \\
\hline Hemithraupis guira (Linnaeus, 1766) & Saíra-de-papo-preto & Guira Tanager & $\mathrm{R}$ & 1,2 & & & u & r \\
\hline Hemithraupis flavicollis (Vieillot, 1818) & Saíra-galega & Yellow-backed Tanager & $\mathrm{R}$ & & & 1,2 & u & o \\
\hline \multicolumn{9}{|l|}{ Emberizidae Vigors, 1825} \\
\hline Ammodramus aurifrons (Spix, 1825) & Cigarrinha-do-campo & $\begin{array}{l}\text { Yellow-browed } \\
\text { Sparrow }\end{array}$ & $\mathrm{R}$ & & & 1,2 & c & O \\
\hline Volatinia jacarina (Linnaeus, 1766) & Tiziu & Blue-black Grassquit & $\mathrm{R}$ & & & 3 & C & O \\
\hline Sporophila lineola (Linnaeus, 1758) & Bigodinho & Lined Seedeater & $\mathrm{R}$ & & & 3 & c & O \\
\hline Sporophila angolensis (Linnaeus, 1766) & Curió & $\begin{array}{l}\text { Chestnut-bellied } \\
\text { Seed-Finch }\end{array}$ & $\mathrm{R}$ & & 1,2 & & u & $\mathrm{P}$ \\
\hline Arremon taciturnus (Hermann, 1783) & Tico-tico-de-bico-preto & Pectoral Sparrow & $\mathrm{R}$ & 1,2 & 1,2 & & c & $r, p$ \\
\hline \multicolumn{9}{|l|}{ Cardinalidae Ridgway, 1901} \\
\hline Habia rubica (Vieillot, 1817) & Tiê-do-mato-grosso & $\begin{array}{l}\text { Red-crowned } \\
\text { Ant-Tanager }\end{array}$ & $\mathrm{R}$ & 1,2 & 1,2 & & u & $r, p$ \\
\hline Cyanoloxia cyanoides (Lafresnaye, 1847) & Azulão-da-amazônia & Blue-black Grosbeak & $\mathrm{R}$ & & 1,2 & & c & $r, p$ \\
\hline \multicolumn{9}{|l|}{$\begin{array}{l}\text { Parulidae Wetmore, Friedmann, Lincoln, } \\
\text { Miller, Peters, van Rossem, Van Tyne \& } \\
\text { Zimmer } 1947\end{array}$} \\
\hline Phaeothlypis fulvicauda (Spix, 1825) & $\begin{array}{l}\text { Pula-pula-de-cauda- } \\
\text { avermelhada }\end{array}$ & Buff-rumped Warbler & $\mathrm{R}$ & 1,2 & 1,2 & & u & $r, p$ \\
\hline Icteridae Vigors, 1825 & & & & & & & & \\
\hline
\end{tabular}

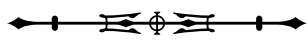


Table 2.

(Conclusion)

\begin{tabular}{|c|c|c|c|c|c|c|c|c|}
\hline Taxon & Portuguese name & English name & St & PC & $M N$ & $\mathrm{GO}$ & CA & TE \\
\hline Psarocolius decumanus (Pallas, 1769) & Japu & Crested Oropendola & $\mathrm{R}$ & 1,2 & 1,2 & & $c$ & $r, p$ \\
\hline Psarocolius bifasciatus (Spix, 1824) & Japuaçu & Olive Oropendola & $\mathrm{R}$ & 1,2 & & & u & 0 \\
\hline Cacicus cela (Linnaeus, 1758) & Xexéu & $\begin{array}{l}\text { Yellow-rumped } \\
\text { Cacique }\end{array}$ & $\mathrm{R}$ & 1,2 & & & $c$ & $r, p$ \\
\hline Lampropsar tanagrinus (Spix, 1824) & Iraúna-velada & Velvet-fronted Grackle & $\mathrm{R}$ & & & 3 & $c$ & $r, p$ \\
\hline Molothrus oryzivorus (Gmelin, 1788) & Iraúna-grande & Giant Cowbird & $\mathrm{R}$ & & & 3 & $c$ & $r, p$ \\
\hline Sturnella militaris (Linnaeus, 1758) & Polícia-inglesa-do-norte & Red-breasted Blackbird & $\mathrm{R}$ & & & 3 & c & $r, p$ \\
\hline \multicolumn{9}{|l|}{ Fringillidae Leach, 1820} \\
\hline Euphonia chlorotica (Linnaeus, 1766) & Fim-fim & Purple-throated Euphonia & $\mathrm{R}$ & & & 2 & c & o \\
\hline $\begin{array}{l}\text { Euphonia laniirostris d'Orbigny \& } \\
\text { Lafresnaye, } 1837\end{array}$ & Gaturamo-de-bico-grosso & Thick-billed Euphonia & $\mathrm{R}$ & & & 1,2 & $\mathrm{u}$ & O \\
\hline Euphonia minuta Cabanis, 1849 & Gaturamo-de-barriga-branca & White-vented Euphonia & $\mathrm{R}$ & 1,2 & 1,2 & & $\mathrm{u}$ & r \\
\hline Euphonia rufiventris (Vieillot, 1819) & Gaturamo-do-norte & $\begin{array}{l}\text { Rufous-bellied } \\
\text { Euphonia }\end{array}$ & $\mathrm{R}$ & 1,2 & & & $\mathrm{u}$ & $P$ \\
\hline
\end{tabular}

described by Stotz et al. (1996) and Hoyo et al. (2003). The species S. ucayalae is considered near threatened by IUCN (2000), and in some cases can be considered as restricted to bamboo forests as well (Guilherme, 2009). We captured this species mostly in sites dominated by bamboos, including second growth and post-burn sites. Despite its near threatened status, S. ucayalae populations may be using these human-influenced environments. It is also relevant to describe the registration of Cnipodectes superrufus, an uncommon bamboo specialist from southwest Amazonia, which may be least abundant and perhaps the most threatened of all bamboo specialists in the Amazonian forests (Lane et al., 2007; Tobias et al., 2008). We captured only one individual in a bamboo/ second growth forest in a post-burned area $\left(10^{\circ} 20^{\prime} \mathrm{S}\right.$, $68^{\circ} 40^{\prime} \mathrm{W}$ ). Finally, it is important to cite five other species registered in our study (Crypturellus atrocapillus, Nonnula ruficapilla, Monasa flavirostris, Lophotriccus eulophotes and Neopelma sulphureiventer) which apparently have a preference of bamboo habitats as well (Kratter, 1997), however, the few registrations in literature and in our field samplings do not permit generalizations
(Table 2). The results of this paper showed that the RESEX Chico Mendes holds a unique Amazonian bird community, influenced by bamboo and second growth vegetation. Although the avifauna described here is far from a complete census of the RESEX, and needs to be resampled in other seasons, the rarefaction curves and estimators indicate a good sampling effort. These data, regarding the local avifauna, reinforce the biological importance of the RESEX and highlight the need for more inventories and bird studies at this isolated and little known region of the Amazon.

\section{ACKNOWLEDGEMENTS}

We thank to local people from Reserva Extrativista Chico Mendes who welcomed and worked with us. We also thank Sidnei Dantas, Alexandre Aleixo and Edson Guilherme who gently helped us to identify the bird songs, pictures and specimens. We also thank to two anonymous referees who significantly helped to improve this manuscript. The data presented here are part of the project "Biodiversity implications of forest disturbance and related landscape dynamics in the Brazilian Amazon"

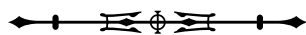


(NNX07AF16G) a collaborative research among South Dakota State University, National Aeronautics and Space Administration, Lancaster University, and Museu Paraense Emílio Goeldi. The Birders Exchange and Idea Wild donated important materials for this research.

\section{REFERENCES}

BIRDLIFE INTERNATIONAL, 2010. IUCN Red List for birds. Species factsheet: Primolius couloni. Available in: <http://www. birdlife.org >. Accessed on: 13 December 2010.

BROWN, I. F., W. SCHROEDER, A. SETZER, M. MALDONADO, M. DE LOS RIOS, N. PANTOJA, A. DUARTE \& J. MARENGO, 2006. Monitoring fires in southwestern Amazonia rain forests. Eos 87(26): 253-264.

CBRO, Comitê Brasileiro de Registros Ornitológicos, 2009. Listas das aves do Brasil. Available in: < http://www.cbro.org.br/CBRO/ index.htm >. Accessed on: 2 December 2009.

COLWELL, R. K., 2004. EstimateS, Version 7: Statistical estimation of species richness and shared species from samples (Software and User's guide). Freeware. Available in:<http://viceroy.eeb.uconn. edu/EstimateSPages/EstSUsersGuide/EstimateSUsersGuide.htm > . Accessed on: 13 December 2010.

COSTA, S. S. M., 2000. Caracterização ambiental da Reserva Extrativista Chico Mendes (Acre-Brasil): subsídios ao plano de manejo: 1-151. Tese (Doutorado em Ecologia) - Universidade Federal de São Carlos, São Paulo. Available in: < http://www.ufmt.br/gpea/ pub/tese-doutorado-suely-de-sousa-melo-da-costa.pdf $>$. Accessed on: 3 December 2010.

ERIZE, F., J. R. R. MATA \& M. RUMBOLL, 2006. Birds of South America, Non passerines: Rheas to Woodpeckers. Princeton University Press, Princeton.

GOTELLI, N. \& R. K. COLWELL, 2001. Quantifying biodiversity: procedures and pitfalls in the measurement and comparison of species richness. Ecology Letters 4(4): 379-391.

GUILHERME, E., 2001. Comunidade de aves do campus e Parque Zoobotânico da Universidade Federal do Acre, Brasil. Tangara 1(2): 57-73.

GUILHERME, E., 2009. Avifauna do estado do Acre: composição, distribuição geográfica e conservação. Tese (Doutorado em Zoologia) - Universidade Federal do Acre/Museu Paraense Emilio Goeldi, Belém, Pará. Available in: <http://www.dominiopublico.gov.br/ download/texto/cp093574.pdf> . Accessed on: 21 November 2010.

HOYO, J. del, A. ELLIOT \& J. SARGATAL, 1994. Handbook of the birds of the world: New world vultures to Guineafowl. Vol. 2. Lynx Edicions, Barcelona.
HOYO, J. del, A. ELLIOT \& J. SARGATAL, 1996. Handbook of the birds of the world: Hoatzin to Auks. Vol. 3. Lynx Edicions, Barcelona.

HOYO, J. del, A. ELLIOT \& J. SARGATAL, 2003. Handbook of the birds of the world: Broadbills and Tapaculos. Vol. 8. Lynx Edicions, Barcelona.

HOYO, J. del, A. ELLIOT \& J. SARGATAL, 2004. Handbook of the birds of the world: Cotingas to Pipits and Wagtails. Vol. 9. Lynx Edicions, Barcelona.

HOYO, J. del, A. ELLIOT \& J. SARGATAL, 2005. Handbook of the birds of the world: Cuckoos - Shrikes to Thrushes. Vol. 10. Lynx Edicions, Barcelona.

INPE, Instituto Nacional de Pesquisas Espaciais, 2010. Monitoramento da floresta amazônica brasileira por satélite. São José dos Campos, SP. Available in: <http://sigma.cptec.inpe.br/queimadas/>. Accessed on: 21 November 2010.

ISLER, M. L., P. R. ISLER \& B. M. WHITNEY, 2007. Species limits in antbirds (Thamnophilidae): the warbling antbird (Hypocnemis cantator) complex. The Auk 124(1): 11-28.

INFONATURA, 2007. Animals and ecosystems of Latin America. Version 5.0. Arlington, Virginia (USA): NatureServe. Available in: <http://www.natureserve.org/infonatura>. Accessed on: 3 November 2010.

IUCN, International Union for Conservation of Nature and Natural Resources, 2000. Protected areas. Benefits beyond boundaries - WCPA in Action: 1-17. Available in: <http://cmsdata.iucn.org/ downloads/wcpainaction.pdf > . Accessed on: 13 December 2010.

KRATTER, A. W., 1997. Bamboo specialization by Amazonian birds. Biotropica 29(1): 100-110.

LANE, D. F., G. P. SERVAT, T. VALQUI \& F. R. LAMBERT, 2007. A distinctive new species of tyrant flycatcher (Passeriformes: Tyrannidae: Cnipodectes) from southeastern Peru. The Auk 124: 762-772.

MESTRE, L. A. M., J. BARLOW, G. THOM \& M. A. COCHRANE, 2009. Burned forests as a novel habitat for the black-faced cotinga (Conioptilon mcilhennyi) in the western Brazilian Amazon. Ornithologia Neotropical 20: 467-470.

MESTRE, L. A. M. \& R. O. B. BIERREGAARD, 2009. The role of Amazonian rivers for wintering ospreys (Pandion haliaetus): clues from North American band recoveries in Brazil between 1937 and 2006. Studies on Neotropical Fauna and Environment 44(3): 141-147.

NELSON, B. W. \& M. N. IRMÃO, 1998. Fire penetration in standing Amazon forests. Proceedings Brazilian Remote Sensing Congress 9: 3-18.

NOVAES, F. C., 1957. Contribuição à ornitologia do noroeste do Acre. Boletim do Museu Paraense Emílio Goeldi, Nova Série Zoologia 9: 1-29. 
NOVAES, F. C., 1958. As aves e as comunidades bióticas no alto rio Juruá, território do Acre. Boletim do Museu Paraense Emílio Goeldi, Nova Série Zoologia 14: 1-13.

PINTO, O. M. O. \& E. A. CAMARGO, 1954. Resultados ornitológicos de uma expedição ao território do Acre pelo Departamento de Zoologia. Papéis Avulsos do Departamento de Zoologia 11(23): 371-418.

RESTALL, R., C. RODNER \& M. LENTINO, 2007. Birds of Northern South America. Yale University Press, Yale.

SICK, H., 1997. Ornitologia brasileira. Nova Fronteira, Rio de Janeiro.

STOTZ, D. F., J. W. FITZPATRICK, T. A. PARKER \& D. K. MOSKOVITS, 1996. Neotropical birds: ecology and conservation. University of Chicago Press, Chicago.

TOBIAS, J. A. \& D. J. BRIGHTSMITH, 2007. Distribution, ecology and conservation status of the blue-headed macaw Primolius couloni. Biological Conservation 139(1-2): 126-138.
TOBIAS, J. A., D. J. LEBBIN, A. ALEIXO, M. J. ANDERSEN, E. GUILHERME, P. A. HOSNER \& N. SEDDON, 2008. Distribution, behavior, and conservation status of the rufous twistwing (Cnipodectes superrufus). The Wilson Journal of Ornithology 120(1): 38-49.

VANZOLINI, P. E., 1952. Relatório de uma expedição cientííca ao Território Nacional do Acre no ano de 1951. Papéis Avulsos do Departamento de Zoologia 11(1): 1-20.

WHITTAKER, A. \& D. C. OREN, 1999. Important ornithological records from the Rio Juruá, western Amazonia, including twelve additions to the Brazilian avifauna. Bulletin of the British Ornithologists' Club 119(4): 235-260.

WHITTAKER, A., D. C. OREN, J. F. PACHECO, R. PARRINI \& J. C. MINNS, 2002. Aves registradas na Reserva Extrativista do Alto Juruá. In: M. CARNEIRO DA CUNHA \& M. B. ALMEIDA (Eds.): Enciclopédia da floresta: $\bigcirc$ Alto Juruá: Práticas e Conhecimentos das Populações: 81-99. Companhia das Letras, São Paulo.

Recebido: 28/02/2010

Aprovado: 20/12/2010

Responsabilidade editorial: Alexandre Aleixo 
